\title{
Immunosuppressive niche engineering at the onset of human colorectal cancer
}

\section{Chandler Gatenbee}

Moffitt Cancer Centre https://orcid.org/0000-0002-9730-5964

\section{Ann-Marie Baker}

https://orcid.org/0000-0001-8905-9137

\section{Ryan Schenck}

H. Lee Moffitt Cancer Center \& Research Institute https://orcid.org/0000-0002-0294-0106

\section{Maximilian Strobl}

H. Lee Moffitt Cancer Center \& Research Institute https://orcid.org/0000-0003-4484-8823

\section{Jeffrey West}

Moffitt Cancer Center https://orcid.org/0000-0001-9579-4664

\section{Margarida Neves}

Barts Cancer Institute, Queen Mary University of London

\section{Sara Hasan}

Barts Cancer Institute, Queen Mary University of London

\section{Eszter Lakatos}

QMUL

\section{Pierre Martinez}

Cancer Research Center of Lyon

\section{William Cross}

Barts Cancer Institute, Queen Mary University London

\section{Marnix Jansen}

University College London https://orcid.org/0000-0003-0645-564X

\section{Manuel Rodriguez-Justo}

University College London https://orcid.org/0000-0001-5007-1761

\section{Christopher Whelan}

Moffitt Cancer Center https://orcid.org/0000-0001-7511-2603

\section{Andrea Sottoriva}

Institute of Cancer Research https://orcid.org/0000-0001-6709-9533

\section{Simon Leedham}

Wellcome Centre for Human Genetics

\section{Mark Robertson-Tessi}

H. Lee Moffitt Cancer Center \& Research Institute https://orcid.org/0000-0003-0143-9587 
Queen Mary University of London https://orcid.org/0000-0001-9582-1597

Alexander Anderson ( $\square$ alexander.anderson@moffitt.org )

H. Lee Moffitt Cancer Center \& Research Institute https://orcid.org/0000-0002-2536-4383

\section{Article}

Keywords: immunosuppressive niche, immunotherapy, Colorectal cancer, tumor initiation

Posted Date: August 18th, 2021

DOI: https://doi.org/10.21203/rs.3.rs-799879/v1

License: (c) (i) This work is licensed under a Creative Commons Attribution 4.0 International License. Read Full License

Version of Record: A version of this preprint was published at Nature Communications on April 4th, 2022. See the published version at https://doi.org/10.1038/s41467-022-29027-8. 


\section{Immunosuppressive niche engineering at the onset of human colorectal cancer}

Chandler D. Gatenbee ${ }^{1}$, Ann-Marie Baker ${ }^{2}$, Ryan O. Schenck ${ }^{1,3}$, Maximilian Strobl ${ }^{1,9}$, Jeffrey West ${ }^{1}$, Margarida P. Neves ${ }^{2}$, Sara Yakub Hasan ${ }^{2}$, Eszter Lakatos ${ }^{2}$, Pierre Martinez ${ }^{2,4}$, William C.H. Cross ${ }^{2}$, Marnix Jansen ${ }^{5}$, Manuel Rodriguez-Justo ${ }^{5}$, Christopher J. Whelan ${ }^{6,7}$, Andrea Sottoriva ${ }^{8}$, Simon Leedham $^{3}$, Mark Robertson-Tessi ${ }^{1 *}$, Trevor A. Graham ${ }^{2 *}$, Alexander R.A. Anderson ${ }^{1 * \dagger}$

${ }^{1}$ Integrated Mathematical Oncology Department, H. Lee Moffitt Cancer Center \& Research Institute, 12902 Magnolia Drive, SRB 4, Tampa, Florida, 336122

2 Evolution and Cancer Laboratory, Centre for Genomics and Computational Biology, Barts Cancer Institute, Queen Mary University of London, London, EC1M 6BQ, UK

${ }^{3}$ Wellcome Centre for Human Genetics, University of Oxford, Oxford, OX37BN, UK.

${ }^{4}$ Lyon Cancer Institute, Lyon, France

${ }^{5}$ Department of Pathology, University College London Hospital, London, UK

${ }^{6}$ Cancer Physiology, H. Lee Moffitt Cancer Center \& Research Institute, 12902 Magnolia Drive, SRB 4, Tampa, Florida, 336122

${ }^{7}$ Department of Biological Sciences, University of Illinois at Chicago, 845 West Taylor Street, Chicago, IL 60607 USA

${ }^{8}$ Center for Evolution and Cancer, Institute of Cancer Research, London, UK

${ }^{9}$ Wolfson Centre for Mathematical Biology, University of Oxford, Oxford, UK

* Senior Author

† Corresponding Author

\section{Author Contributions}

CDG developed and analyzed the computational model, developed the methods to segment and align the immunohistochemistry, and performed analysis of the data collected from immunohistochemistry and neoantigen predictions. AMB designed, optimized and performed all laboratory experiments, with the assistance of MPN and SYH. ROS processed whole exome sequencing of samples and downstream data generation and WCHC, PM, EZ and AS provided additional support in genomic analysis. MRJ, SL and AS graciously provided samples and/or data. MRJ and MJ provided histopathological assessment. CJW provided valuable feedback on the ecological analysis. MRT, TAG, and ARAA guided the research and supervised the writing of the manuscript. TAG and ARAA conceived and funded the study. 


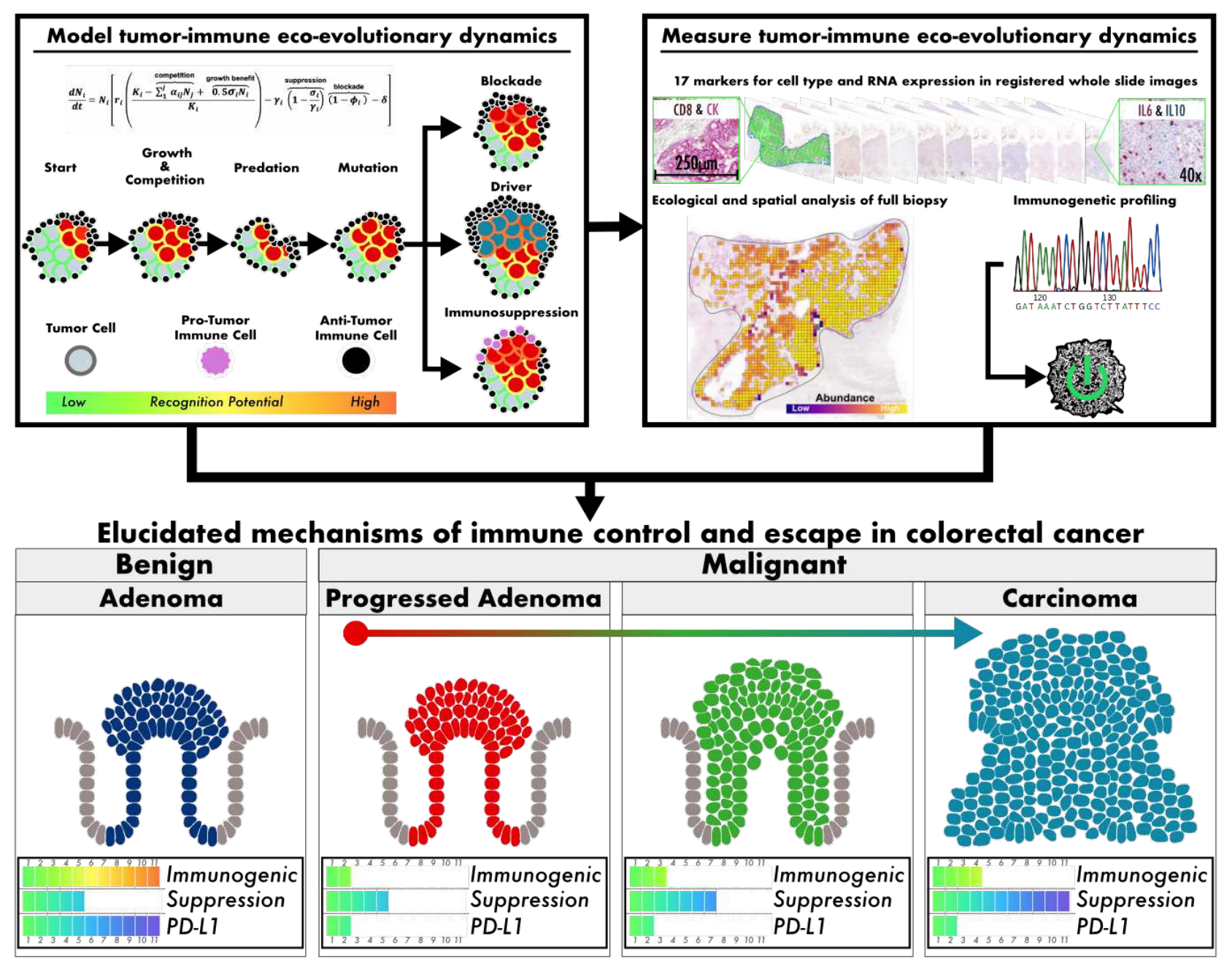

\section{Abstract}

The evolutionary dynamics of tumor initiation remain undetermined, and the interplay between neoplastic cells and the immune system is hypothesized to be critical in transformation. Colorectal cancer (CRC) presents a unique opportunity to study the transition to malignancy as pre-cancers (adenomas) and earlystage cancers are frequently detected and surgically removed. Here, we demonstrate a key role for the immune response in tumor initiation by studying tumor-immune eco-evolutionary dynamics from pre-cancer to carcinoma using a computational model, ecological analysis of digital pathology data, and multi-region exome sequencing and neoantigen prediction in a total of 62 patient samples. Modelling indicates there are several potential routes to malignancy, each of which uniquely sculpts tumor ecology and intra-tumor antigenic heterogeneity (alTH). In patient samples, the immune microenvironment was characterized using the spatial distribution of 17 markers across registered whole-slide images, as well as patterns of intralesion alTH measured using multi-region exome sequencing and neoantigen prediction. The patient data were best described by a model where adenomas that become immunogenic early on do not progress to CRC because they are under immune control; progression therefore proceeds in adenomas with low immunogenicity. In these tumors, immune suppression is initially low, but gradually an immunosuppressive niche that is depleted in CD8+ cytotoxic T cells expands. There was little evidence for immune blockade (PD-L1 expression) in tumor initiation or progression. These results suggest that re-engineering the immunosuppressive niche may prove to be an effective immunotherapy in CRC. 


\section{Introduction}

The classical model of colorectal carcinogenesis is the adenoma-carcinoma pathway that describes the accumulation of (epi)mutations in benign (non-invasive) adenomas, which underpin the development of invasive carcinoma (Kinzler \& Vogelstein, 1996; Muto, Bussey, \& Morson, 1975). However, while the risk of developing colorectal cancer (CRC) is certainly increased by adenoma formation (Carvajal-Carmona et al., 2013; Zauber et al., 2012), it appears that few adenomas actually progress to cancer in a human lifetime: bowel cancer screening programs detect approximately five 'high risk' adenomas for every cancer found (Logan et al., 2012; Zauber et al., 2012), and longitudinal endoscopic surveillance of adenomas reveals that less than $2 \%$ of adenomas progress to cancer within three years (Hofstad et al., 1996). Consequently, there appears to be a substantial 'evolutionary hurdle' that must be overcome for an adenoma to become invasive.

Immune predation is known to modulate and indeed suppress neoplastic growth (Grivennikov, Greten, \& Karin, 2010). As in lung cancer (Mascaux et al., 2019; Rosenthal et al., 2019), the evolution of immune evasion is therefore likely to be a key barrier on the evolutionary path to CRC. Newly arising somatic mutations in a tumor may generate neoantigens, which can then serve as targets for immune-cell recognition and destruction (in particular CD8+ cytotoxic T lymphocytes). However, the negative selective pressure imposed by the immune system provides a positive selection pressure for strategies to avoid elimination, a process known as immunoediting (Dunn, Bruce, Ikeda, Old, \& Schreiber, 2002; Grasso et al., 2018). Such immune evasion has been described as a hallmark of cancer (Hanahan \& Weinberg, 2011), and there are many mechanisms by which tumor cells may escape immune predation including, but not limited to, blockade of cytotoxic T-cell attack via expression of programmed death-ligand 1 (PD-L1), recruitment of immunosuppressive cells such as macrophages and neutrophils, and disruption of the antigen presentation machinery (Coffelt, Wellenstein, \& de Visser, 2016; Khong \& Restifo, 2002; McGranahan et al., 2017; Schreiber, Old, \& Smyth, 2011; Vinay et al., 2015).

In CRC, multiple lines of evidence suggest a critical role for immunological surveillance in regulating tumor growth. The density of tumor-infiltrating $T$ cells is highly prognostic, with greater infiltration associated with a better prognosis (Galon et al., 2006; Pages et al., 2009). Moreover, non-metastatic CRC have an increased level of T-cell infiltration as compared to metastatic CRC (Pages et al., 2005). Genomic analysis reveals that a higher predicted neoantigen burden is associated with increased tumor lymphocyte infiltration (Angelova et al., 2015; Giannakis et al., 2016; Rosenthal et al., 2019). Large scale genomic analysis indicates immune evasion mechanisms have evolved in the majority of CRC (Grasso et al., 2018). Immune modulation studies in mouse models of $\mathrm{CRC}$ also provide support for a critical regulatory role of the immune system in colorectal carcinogenesis (Kim et al., 2006; Ngiow et al., 2011; Yu, Steel, Zhang, Morris, \& Waldmann, 2010).

The progression from colorectal adenoma (CRA) to CRC likely requires the accumulation of multiple (epi)genetic aberrations. The overall single nucleotide alteration (SNA) burden appears comparable between CRA and CRC, including SNAs for putative driver genes, with the exception of TP53 (Cross et al., 2018). Moreover, analysis of the evolutionary dynamics of sub-clones within CRC indicates a frequent lack of differential selection operating between subclones (Williams et al., 2018), suggesting that at the point of invasion, the founder cancer cell had already acquired all the alterations necessary for its malignant phenotype and also that the bulk of tumor cells were not experiencing markedly differential immune predation. Thus, malignant potential and immune evasion may be established together when cancer growth is initiated, and conceivably immune evasion could be the key phenotypic trait governing transition from adenomas to cancers.

By integrating mathematical modeling, ecological analysis of whole slide images, and multi-region neoantigen prediction, here we have investigated the role of immune escape in the evolution of CRC from precursor CRA. We hypothesized that immune surveillance represents a key hurdle that prevents the outgrowth of invasive cells within a benign CRA. For the neoplastic cells, the acquisition of mutations responsible for progression must be balanced against the risk of accumulating too many neoantigens that would lead to immune elimination. To investigate this idea, we developed a mathematical model that simulates tumor evolution under immune predation and escape in order to define the expected patterns of 
immune activity and antigenic intratumor heterogeneity (alTH) throughout tumor progression from benign to malignant. We then looked for these signatures in a cross-sectional cohort of CRA as well as early and later stage CRC using the expression of 17 markers (by immunohistochemistry (IHC) and RNA in situ hybridization (ISH)) and called neoantigens from multi-region whole exome sequencing (WES) data. We leveraged several ecological tools to describe and compare the cellular composition of tumors as biological units, providing a holistic view of how tumors change through progression. Comparison of the model and data indicates a key role for immune evasion at the onset of malignancy in colorectal cancer.

\section{Results}

\section{Immune suppression is the superior escape strategy}

A

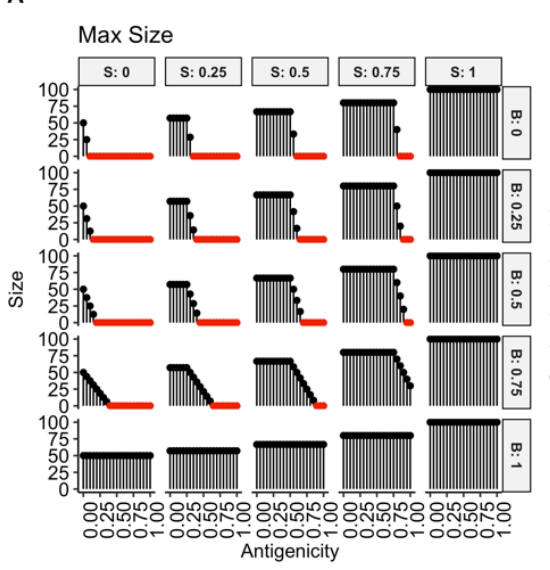

B

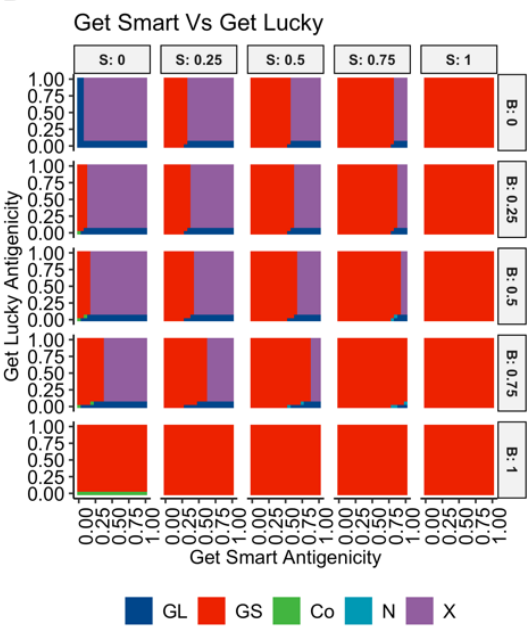

C

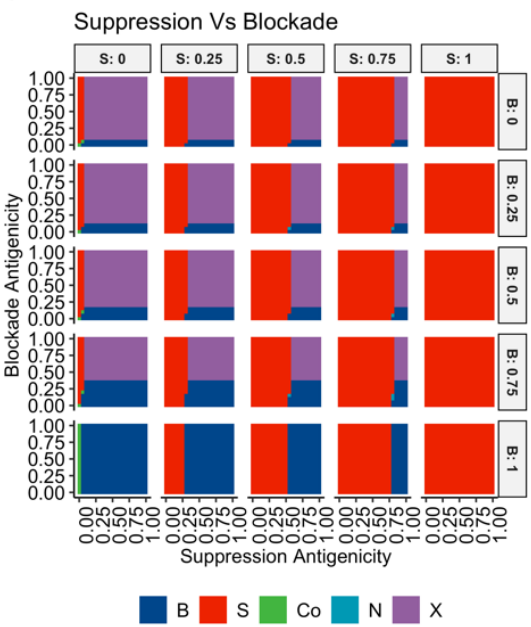

Figure 1. Model results predict that immune suppression is the dominant escape mechanism. A) Maximum size (i.e. number of cells) of each strategy for different values of suppression ( $\mathrm{S}, \sigma$, columns), blockade (B, $\phi$, rows), and antigenicity (inset $\mathrm{x}$-axes). Red dots indicate that the population is eliminated by immune predation, highlighting that clones must have some way to avoid immune predation, either by having low antigenicity (Get Lucky) or actively mitigating attack (Get Smart). As population growth was simulated using an epithelial division rate, these results indicate immune escape should be an early event if the clone is to survive increased predation due to the accumulation of neoantigens associated with mutation. B, C) Outcome of competition, where "Co" means neither strategy would go extinct and could co-exist, "N", indicates either strategy could win, but the one that does must have a larger starting population size, and "X" means that neither population survived immune predation. B) Outcomes of competition between Get Lucky $(\mathrm{GL})$ and Get Smart (GS), over a range of suppression values (S, $\sigma)$, blockade values $(\mathrm{B}, \phi)$, and antigenicities $(\gamma)$, such that for $\mathrm{GL} \sigma=0, \phi=0$, and for GS $0 \leq \phi \leq 1,0 \leq \phi \leq 1$. Get Smart wins out over Get Lucky $71 \%$ of the time (7778 out of 11025 parameter combinations). C) Outcomes of competition between Suppression (S) and Blockade (B), over a range of suppression values (S, $\sigma$ ), blockade values $(\mathrm{B}, \phi)$, and antigenicities $(\gamma)$, such that for $\mathrm{P} \sigma=0,0 \leq \phi \leq 1$, and for $\mathrm{S} \phi=0,0 \leq \sigma \leq 1$. Out of the 11025 parameter combinations, Suppression (S) wins the majority of the time, $55 \%$ (6047 parameter combinations), with Blockade (B) winning $17 \%$ of the time (1862 parameter combinations). Combined, these results highlight that Get Smart almost always wins out over Get Lucky, while Suppression wins against Blockade. This indicates that having an immune escape strategy (Get Smart), particularly immune suppression, significantly increases a clone's fitness, allowing it to sweep through the population, often by engineering an immunosuppressive niche.

Ecologists have long used mathematical models to simulate the dynamics of interacting species. LotkaVolterra models, in particular, have been used to study predator-prey dynamics, competition, mutualism, and amensalism (May, 1976; J. D. Murray, 2002; Wangersky, 1978). Such models have frequently been adopted by mathematical oncologists to study the evolution of resistance under different therapeutic regimes, as well as tumor-immune interactions (Agarwal \& Bhadauria, 2013; Babbs, 2012; Gatenby \& Vincent, 2003; Strobl et al., 2020). Here, we combine various forms of deterministic Lotka-Volterra models to understand the role of the immune system in tumor initiation and progression under immune predation and subsequent escape from immune control via two distinct "strategies". The first strategy, Blockade, gives tumor cells the ability to effectively neutralize cytotoxic $T$ cells by blocking their attack. Two biological examples of Blockade would be PD-L1 and PI-9, the first of which inhibits cytotoxic T cells (Topalian, Drake, \& Pardoll, 2015), while the latter inhibits cytotoxic T-cell-induced apoptosis by blocking the 
perforin/granzyme B pathway (Medema et al., 2001). The second strategy, Suppression, gives tumor cells the ability to recruit immunosuppressive cells, such as M2 macrophages. Many of these immunosuppressive cells are also involved in wound repair, and thus create a microenvironment that not only suppresses anti-tumor inflammation, but one that also promotes cellular growth via angiogenesis, production of growth factors (e.g. EGF), matrix metalloproteinases (Alberto Mantovani, 2014; A. Mantovani, Biswas, Galdiero, Sica, \& Locati, 2013; Wynn, Chawla, \& Pollard, 2013)

In our Lotka-Volterra model of competition, predation, mutualism, and amensalism, tumor cells compete with one another, are preyed upon by cytotoxic $T$ cells, and are supported by "mutualist" immunosuppressive cells that suppress immune attack and promote growth:

$$
\frac{d N_{i}}{d t}=N_{i}[r_{i}\left(\frac{K_{i}-\overbrace{\sum_{1}^{j} \alpha_{i j} N_{j}}^{\text {competition }}+\overbrace{0.5 \sigma_{i} N_{i}}^{\text {growth benefit }}}{K_{i}}\right)-\gamma_{i} \overbrace{\left(1-\frac{\sigma_{i}}{\gamma_{i}}\right)}^{\text {suppression }} \overbrace{\left(1-\phi_{i}\right)}^{\text {blockade }}-\delta] \quad \text { Equation } 1
$$

Each $i^{\text {th }}$ subpopulation of tumor cells, with population size $N_{i}$, is composed of cells that have four unique traits:

1) Antigenicity $\left(\gamma_{i}\right)$, which defines the immune kill rate and is determined by the collection of neoantigens carried by the cells in the $i^{\text {th }}$ subpopulation (Coulie, Van den Eynde, van der Bruggen, \& Boon, 2014);

2) Degree of Blockade $\left(\phi_{i}\right)$, a cell-intrinsic mechanism that reduces the effective killing rate by cytotoxic Tcells on the $i^{\text {th }}$ subpopulation;

3) Degree of Suppression $\left(\sigma_{i}\right)$, which determines the ability of tumor cells to recruit immunosuppressive cells; this has a dual effect of reducing immune kill and enhancing growth for the $i^{\text {th }}$ population (reviewed in (Coffelt et al., 2016; Khong \& Restifo, 2002; Schreiber et al., 2011; Vinay et al., 2015)); and

4) Species $(j)$, determined by the number of driver mutations accumulated by each cell in the stochastic model. Populations with less than 2 driver mutations are "normal"; those with 2 or 3 mutations are adenomas (CRA), while those with 4+ mutations are carcinomas (CRC). The species of the population determines the population's division rate, carrying capacity, and interactions with other tumor populations (see next section for more details).

Each subpopulation has a distinct carrying capacity $\left(K_{i}\right.$, the maximum viable size of the subpopulation), division rate $\left(r_{i}\right)$, and interactions with other species, defined in the interaction matrix, $\boldsymbol{\alpha}$. We assume that all subpopulations have the same intrinsic death rate, $\delta$.

In simulations of the model, two distinct trajectories emerged. The first trajectory, which we dub "Get Lucky", is when tumor cells acquired only mutations that had low antigenicities, but lacked an active escape mechanism $\left(\sigma_{i}=0, \phi_{i}=0\right)$ (Figure 1A, top left panel). The second trajectory, termed "Get Smart", is when tumors acquired one or more mutations that facilitated escape from elimination by the immune system $\left(\sigma_{i}>\right.$ 0 and/or $\phi_{i}>0$ ); this in turn reduced the selection pressure against high antigenicity mutations (Figure 1C). These two trajectories demonstrated that it was necessary for tumor cells to mitigate immune attack if they were to grow. Cells that did not avoid immune predation (i.e. having antigenicity too high and having no/weak escape mechanisms) were always eliminated (red dots in Figure 1A).

Tumors are heterogeneous, and it is likely that cells following the Get Smart and Get Lucky trajectories would, at some point, co-exist in a single tumor. To determine the outcome of Get Lucky versus Get Smart competition, which would define the tumor's susceptibility to immune attack (and thus immunotherapies), we conducted a steady state analysis of the pairwise competition between the Get Lucky strategy $\left(\sigma_{i}=\right.$ $0, \phi_{i}=0$ ), and the range of possible Get Smart strategies (combinations of $\sigma_{i} \geq 0, \phi_{i} \geq 0$ ) (Figure 1B). This competition was conducted over a range of antigenicities $\left(0 \leq \gamma_{i} \leq 1\right)$ for each strategy, which allowed us to examine the role of the immune response in determining which strategy would win and which would be 
eliminated. We assumed that both strategies existed within the same 'species' of tumor cell, and thus had the same division rate, shared a carrying capacity, and, aside from the strategy, neither population had any intrinsic advantage over the other (i.e., $\alpha_{i j}=1$ ).

The Get Lucky versus Get Smart analysis indicated that out of the 11025 parameter combinations, in $71 \%$ of the simulations Get Smart out-competed Get Lucky, regardless of the starting sizes of each population. Get Lucky only won 3.3\% of the time, which occurred only when the competing Get Smart population's protection was insufficient to overcome its elevated antigenicity. Given that coexistence was rare $(0.25 \%$ of simulations), these results suggest that clones which have the ability to escape immune attack (Get Smart) will sweep through the population, resulting in the extinction of immune-susceptible clones.

Given that Get Smart is expected to almost always outcompete Get Lucky, we next determined under which inflammatory conditions each individual escape strategy, Blockade or Suppression, could (co)exist. Suppression populations were modeled by setting $0 \leq \sigma_{i} \leq 1$ and keeping $\phi_{i}=0$, while Blockade populations were modeled by setting $0 \leq \phi_{i} \leq 1$ and keeping $\sigma_{i}=0$. By conducting steady-state analyses over all combinations of $\sigma_{i}, \phi_{i}, \gamma_{i}$ (each varying between 0 and 1 in increments of 0.05 ), we could thus determine the outcome of all pairwise competition models of Blockade versus Suppression, each subject to their own immune pressures as determined by their antigenicity.

The Blockade versus Suppression analysis revealed that immune suppression was the superior strategy, as it outcompeted Blockade in the majority of simulations (55\%, 6047 out of 11025 simulations) due to the ability to both increase growth and reduce immune kill rates, which resulted in a higher net growth rate (Figure 1D \& E, and Supplemental Figure S1). Only rarely did initial population size matter (0.054\% of simulations, Figure 1C), as Suppression won frequently even when it had a smaller starting size than Blockade. As in the Get Lucky versus Get Smart analysis, coexistence between the individual strategies was rare $(0.25 \%)$. Together, these observations suggest that clones which can recruit immunosuppressive cells should sweep through the population, even outcompeting cells capable of blocking immune attack directly.

These modelling results suggest that active immune escape mechanisms (as opposed to passive escape by getting lucky) should frequently be observed in established cancers, and that the recruitment of immunosuppressive cells that also increase tumor cell viability is expected to be common among successful tumors.

\section{Immune suppression expected to occur early, increase risk, and shape intra-tumor antigenic heterogeneity}

A

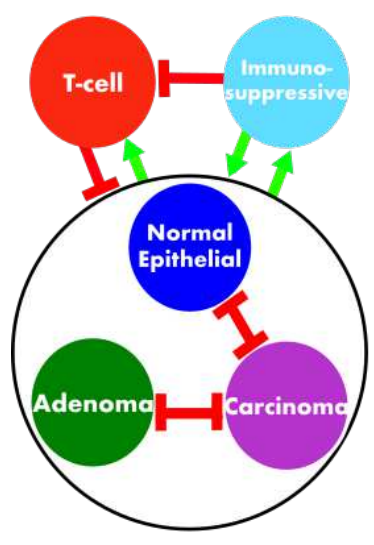

B

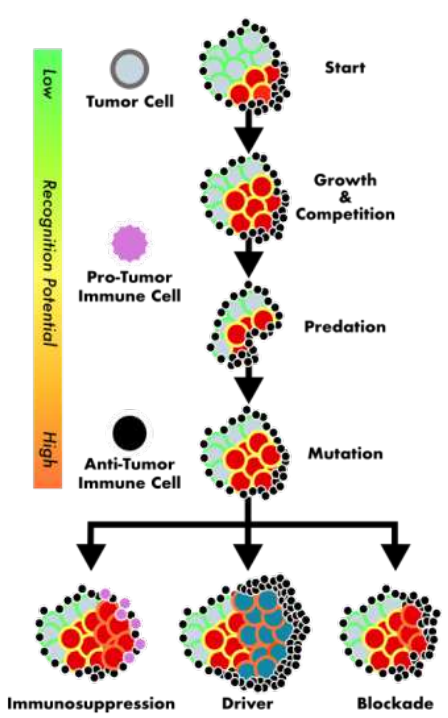

C
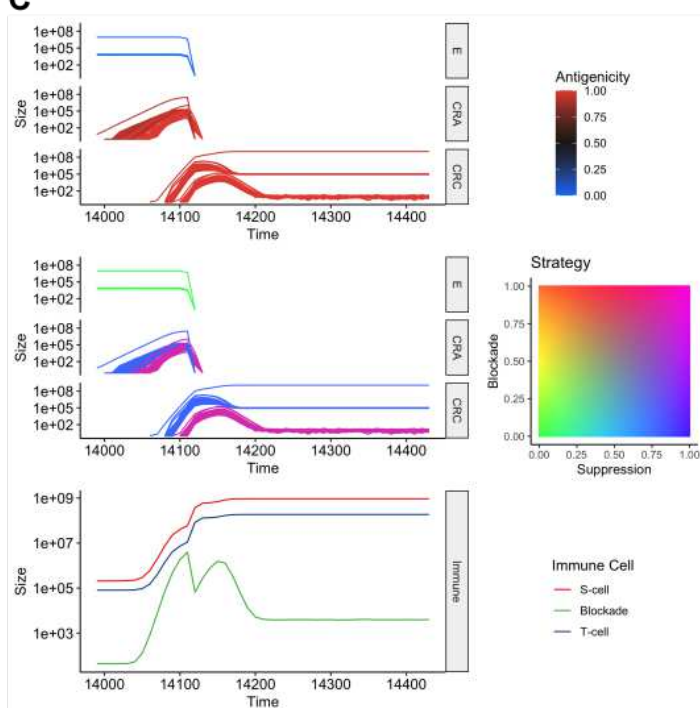
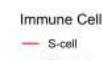

- Blockade

- Tlockill

Figure 2. The competition model in Equation 1 was expanded to include mutation, allowing for simulation of evolution from pre-tumor epithelium (E) to adenoma (CRA) to carcinoma (CRC), with the possibility to acquire driver mutations and immune escape strategies (blockade or suppression). A) This model simulates cell-cell competition, predation by cytotoxic T-cells, mutualism between tumor 
cells and immunosuppressive cells, and reduction of T-cell infiltration by immunosuppressive cells (amensalism). Red bars indicate inhibitory interactions while green arrows represent positive interactions. B) The model is initiated with a large population of homogenous non-immunogenic epithelial cells. Mutation may occur during division, resulting in the creation of a new population that inherits all ancestral antigens and mutations from its parent. Each mutation is accompanied by the generation of a neoantigen, which stimulates increased attack by cytotoxic T cells at a rate proportional to the neoantigen's randomly assigned recognition potential. With low probability, cells may also acquire one of three beneficial mutations: 1) the ability to recruit immunosuppressive cells, which decreases T-cell attack while increasing growth rates; 2 ) the ability to block T-cell attack, reducing immune kill; and 3) acquisition of driver mutations, which increase division rates and carrying capacities once enough have accumulated. If an epithelial population acquires two driver mutations it is considered an adenoma, which grows in a separate niche atop the epithelial tissue, limiting interactions between the two populations. CRA become carcinomas when they acquire four driver mutations in total. CRC have the ability to grow both atop and into the epithelium, allowing carcinomas to invade and destroy epithelial and adenoma clones. Due to inheritance, there may eventually be clones that accumulate multiple beneficial mutations. C) Example simulation, where an $\mathrm{E}$ population evolved into a CRA after 1390 days, which in turn evolved into a CRC. The dominant CRC phenotype had a high antigenicity (top) and strong immunosuppression (middle), resulting in an increase in immunosuppressive cells and a drop in T cells (bottom). Over 200,000 phenotypes developed in this simulation, but only those populations with greater than 50,000 cells are shown.

While deterministic Lotka-Volterra models are readily tractable, they are limited in that they simulate interactions between a fixed number of species, meaning that they cannot simulate the evolution of novel clones, nor generation of intra-tumor heterogeneity. In order to simulate the emergence and subsequent evolution of tumor clones, we created an evolutionary branching process version of the model, which allows us to determine the timing of immune escape, how much it increases the risk of progression to cancer, and how the ecology and alTH changes through progression of CRC. We also simulate the accrual of somatic mutations (see supplemental methods), the evolution through 'tissue compartments' (from healthy tissue (E) into an adenoma (CRA) and then carcinoma (CRC)), and explicitly represent the interactions with immune cells (Figure 2A).

Simulations are initiated with a homogenous, non-antigenic epithelial population that lacks immune-escape mechanisms. Each cell acquires new antigenic mutations at rate $\mu$ per division, and strict inheritance of mutations means a new mutant daughter cell (and its subsequent clone) will always be more antigenic than its parent, since it carries all parental mutations along with the new mutation. Cells can also acquire the ability to block immune attack $\left(\phi_{i}>0\right)$, recruit immunosuppressive cells $\left(\sigma_{i}>0\right)$, or gain a single driver mutation at per-division rates of $3.7 \mathrm{e}-6$ for blockade and suppression, and $9.26 \mathrm{e}-5$ for gaining a driver gene (Figure 2B, and see Supplemental materials for details on calculation of mutation rates). We assumed an epistatic model of progression (Fearon \& Vogelstein, 1990), such that the acquisition of two driver mutations defined the transition from $E$ to CRA, and the acquisition of a further two driver mutations defined the transition to CRC. We modelled competition among species based on the space they occupied: $E$ and CRA clones have no interactions because adenomas grow superficially to the epithelium, whereas we assumed that $C R C$ subpopulations were strongly interacting with both $E$ and CRA populations to describe overgrowth of the adenoma and invasion through the epithelium (Figure 2A).

We assume that the number of immunosuppressive cells will be proportional to the product of the immunosuppressive cell recruitment parameter and the size of the immunosuppressive cell-recruiting population $\left(N_{i} \sigma_{i}\right)$. Likewise, we assume that the number of cytotoxic $\mathrm{T}$ cells reactive to a subpopulation would be proportional to the subpopulation size, its antigenicity, and ability to recruit immunosuppressive cells, $N_{i} \gamma_{i}\left(1-\frac{\sigma_{i}}{\gamma_{i}}\right)$. We can therefore calculate the number of immunosuppressive cells, cytotoxic T cells, and number of cells with immune blockade. Figure $2 \mathrm{C}$ shows a representative simulation run of the model.

In order to understand the role of immune blockade and suppression in determining the risk of cancer development, we conducted a parameter sweep over the ranges of blockade strength $(\phi)$ and suppression strength $(\sigma)$. This also allowed us to see how each strategy affects alTH and also when they emerge in the timeline of cancer progression. The sweeps ranged from 0 to 1 by increments of 0.04 for each of the two parameters. A mutation rate of 2.91e-9 was used in accordance with measurements from Werner et al. (Werner et al., 2020). We furthermore conducted 100 runs of each parameter set to be able to explore the effects of the stochasticity in the model.

There were four possible outcomes of each simulation: (1) The tissue remained healthy for 100 years, which occurred when either no CRA/CRC populations evolved, or remained under 100 cells in size if they did evolve; (2) a CRA evolved but was eliminated; (3) a CRC evolved but was eliminated; (4) a CRC evolved 
from a CRA, and the CRC existed for 1 year, thus considered malignant. In Cases 2 and 3, the simulations were concluded after these elimination events, and the time was recorded.

As we observed in the previous model (Figure 1), the Get Smart strategy dominated the CRC outcome in this expanded model. We examined the immune escape status of these populations in more detail, using a total 21,723 simulations where a CRC formed. Recall there are 3 Get Smart strategies (immunosuppression, blockade, or immunosuppression + blockade), and in $75.87 \%$ of those simulations, the ability to suppress immune attack was an early event; immunosuppression was a trait of the precursor CRA, meaning that the first CRC cell arising from a driver mutation already possessed the immunosuppressive phenotype (Figure 3A). When the precursor CRA relied on a Get Lucky strategy, the emerging $\mathrm{CRC}$ was founded by a clone with the ability to suppress immune attack in $8.72 \%$ of simulations. Across all parameter combinations, only $1.45 \%$ of CRC (and thus their pre-cursor CRA) were successfully established without active immune escape (i.e. these tumors had completely followed the "Get Lucky" trajectory).

Greater immune suppression, which resulted from stronger recruitment of anti-inflammatory pro-growth immune cells, significantly increased the risk and rate of progression from CRA to CRC (Figure 3B,C). In contrast, no matter its strength, the ability to reduce immune kill through increased blockade had little effect on risk and rate of progression. Tumors also became more antigenic as immune suppressive mechanisms became more effective. This phenomenon occurs because immune suppression relaxes selection against high antigenicity, allowing more antigenic mutations to persist (Figure 3D).

A

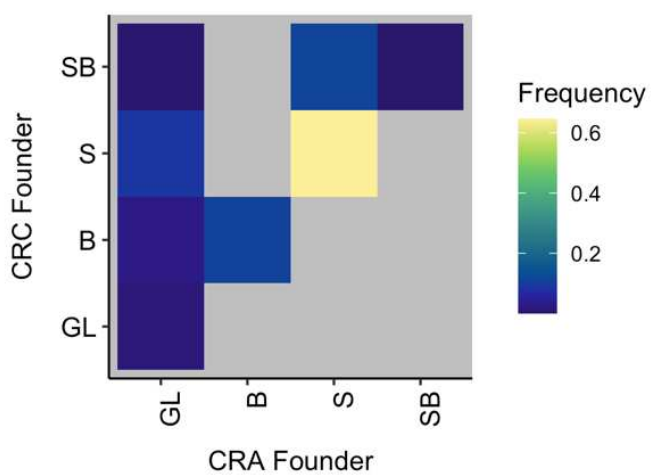

C

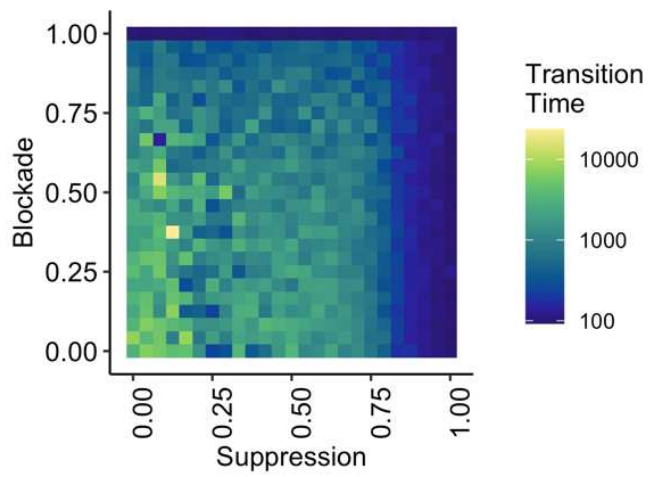

B

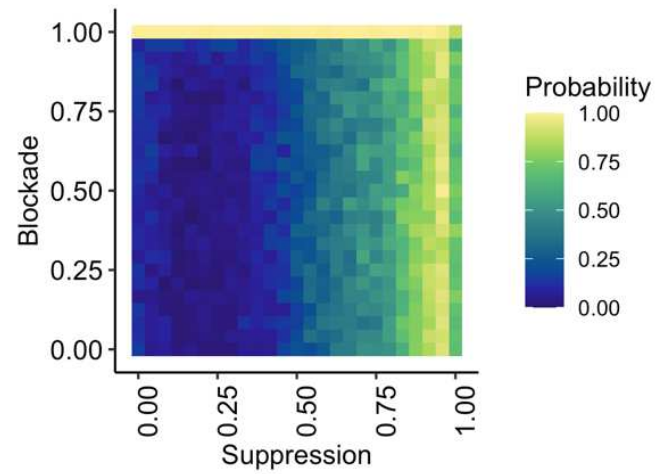

D

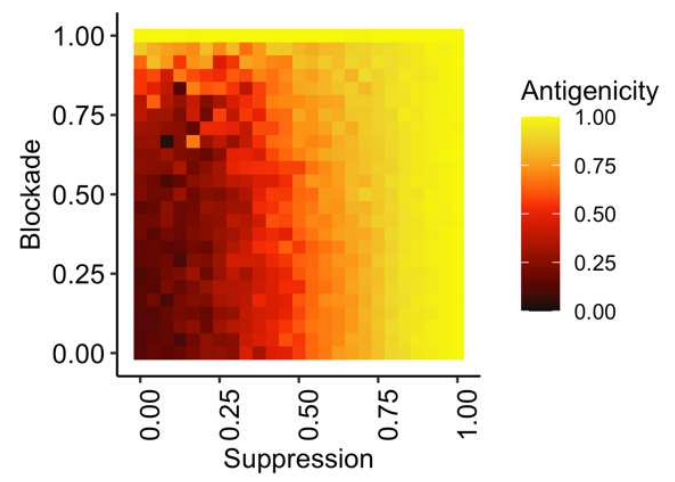

Figure 3. Immune escape occurs early and increases risk of tumor formation. A) Comparison of the CRA founder's immune escape phenotype to the eventual founding branch of the CRC population, where GL = founder relied on Get Lucky, B = founder had Blockade, $\mathrm{S}=$ founder had Suppression, and SB = founder had Suppression and Blockade. Out of the 21,723 simulations, $75.87 \%$ of the simulations had acquisition of Suppression as an early event, occurring in the CRA founder and later inherited by the CRC founder. B) The probability of a carcinoma forming under various strengths of immune escape. Probability is calculated as the number of times a carcinoma existed for 1 year, divided by the number of times that parameter set was run, in this case 100 times each. The gradient from left to right indicates that immune suppression increases the probability of tumor formation, while lack of a gradient from top to bottom suggests that immune blockade has little effect on the probability. C) The average amount of time, in days, between CRA and $\mathrm{CRC}$ formation, i.e. the transition time, for each parameter set. Tumors that progressed most rapidly from CRA to CRC are those with 
an immunosuppressive strategy, which reduces immune predation and relaxes selection against high antigenicity. D) Averages of each malignant tumor's mean antigenicity (weighted by each population's size) for each parameter combination. The horizontal gradient indicates that immune suppression allows for increased antigenicity, as immune suppression relaxes selection against immunogenicity.

\section{Immunosuppressive niche construction begins early in colorectal cancer}

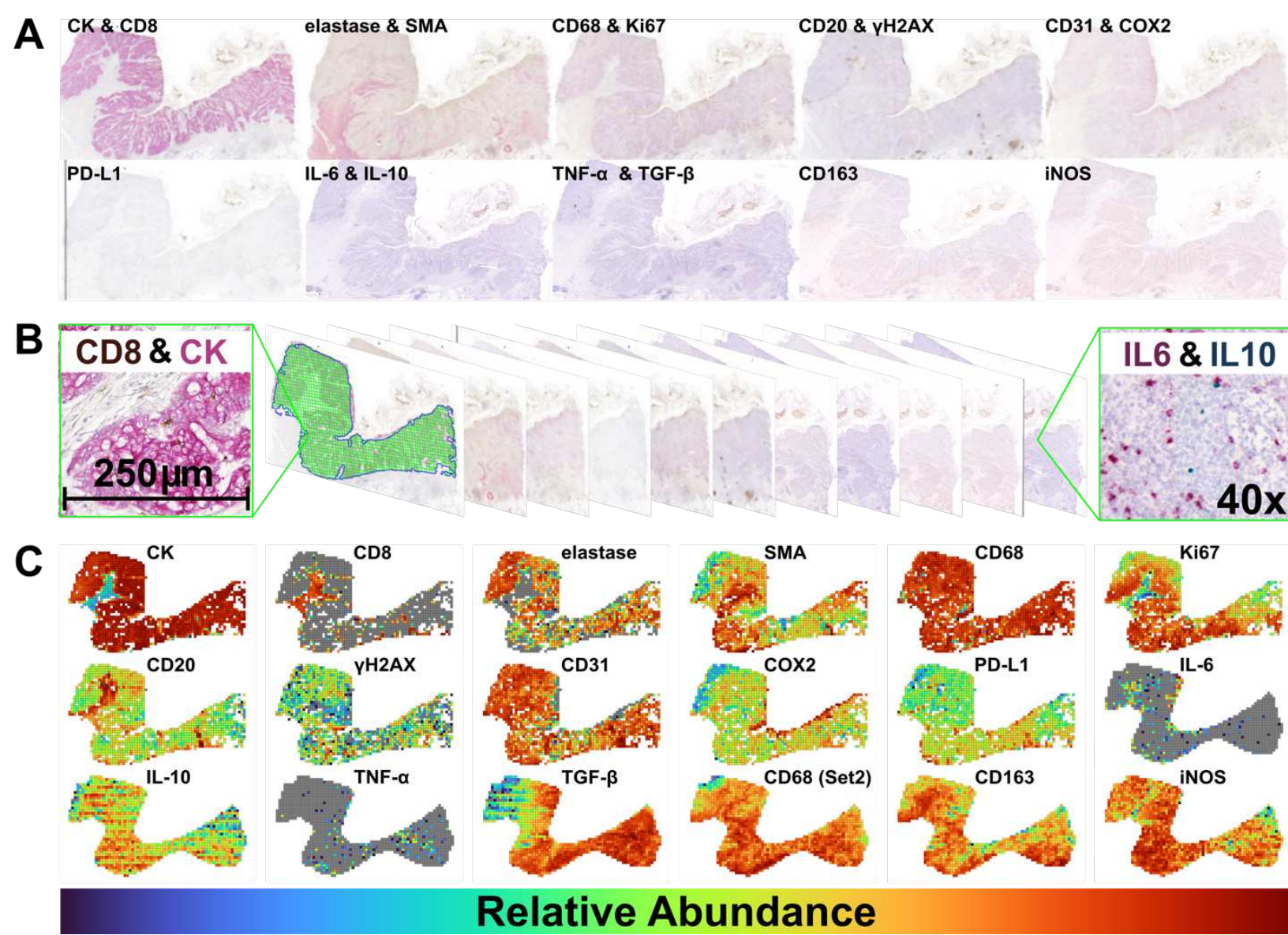

Figure 4. Example of a single sample used to describe the tumor ecology. A) To describe the spatial distribution of cell types and gene expression, 10 serial slices were taken from each sample and each stained with one or two markers. There were two sets of serial slices, one with CK, CD8, elastase, SMA, CD68, Ki67, CD20, $\gamma \mathrm{H} 2 \mathrm{AX}, \mathrm{CD} 31$, COX2, and PD-L1. The second set included IL-6, IL-10, TNF- $\alpha$, TGF- $\beta$, CD163, CD68, and iNOS. B) After aligning the images in the two sets, each slice was divided into quadrats of $250 \mu \mathrm{m} \times 250 \mu \mathrm{m}$, and stain segmentation was performed to determine the abundance of each marker at 40x magnification. C) Quadrat counts for all 18 markers in one CRC sample (there are 17 unique markers, but CD68 is in both Set 1 and Set 2). This process was repeated with 21 colorectal adenomas (CRA), 15 colorectal carcinomas (CRC), and 25 "carcinoma-in-adenoma" (CIA) samples, allowing us to describe changes in the tumor ecology, cell abundance/gene expression, and spatial associations. Values in each plot are scaled to reflect the minimum and maximum values of that marker in the image. See Figure S3 for more detailed images of each marker, and Figures S4-S6 for figures showing quadrat counts for all samples.

We next examined primary human tumors to determine the dominant escape trajectory in CRC, and the time at which it emerged, using model predictions to guide the analysis. Specifically, we considered 21 colorectal adenomas (CRA), 15 colorectal carcinomas (CRC), and 25 "carcinoma-in-adenoma" (CIA) samples, that latter of which consist of a nascent carcinoma (C-CIA) adjacent to an adenoma (A-CIA) (Figures 4-7). As such, we consider the A-CIA samples as progressed/late adenomas, as they are likely either the precursor of the adjacent carcinoma, or in a similar state as that carcinoma's actual precursor.

We performed multi-color immunohistochemical analysis to characterize the immune microenvironment (tumor ecology) in all $n=86$ cases ( 61 samples, with each of the $25 \mathrm{CIA}$ samples having both an A-CIA and 
C-CIA region) (Figure 4, and Supplemental Figure S2-S6). We used multi-region whole exome sequencing (WES) to measure neoantigen intra-tumor heterogeneity (alTH) in a subset of $n=16$ cases (160 exomes, as each was downsampled 10 times to normalize depth).

\section{Adenomas and carcinomas have distinct hot and cold ecologies}

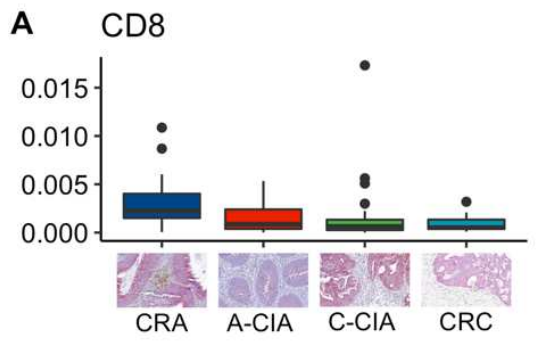

D

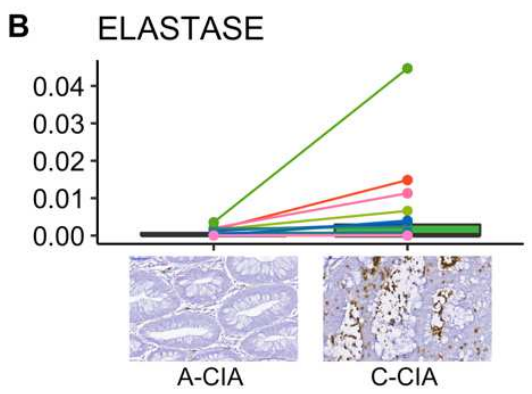

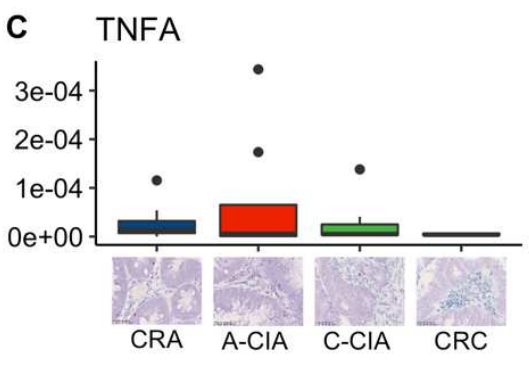

Abundance
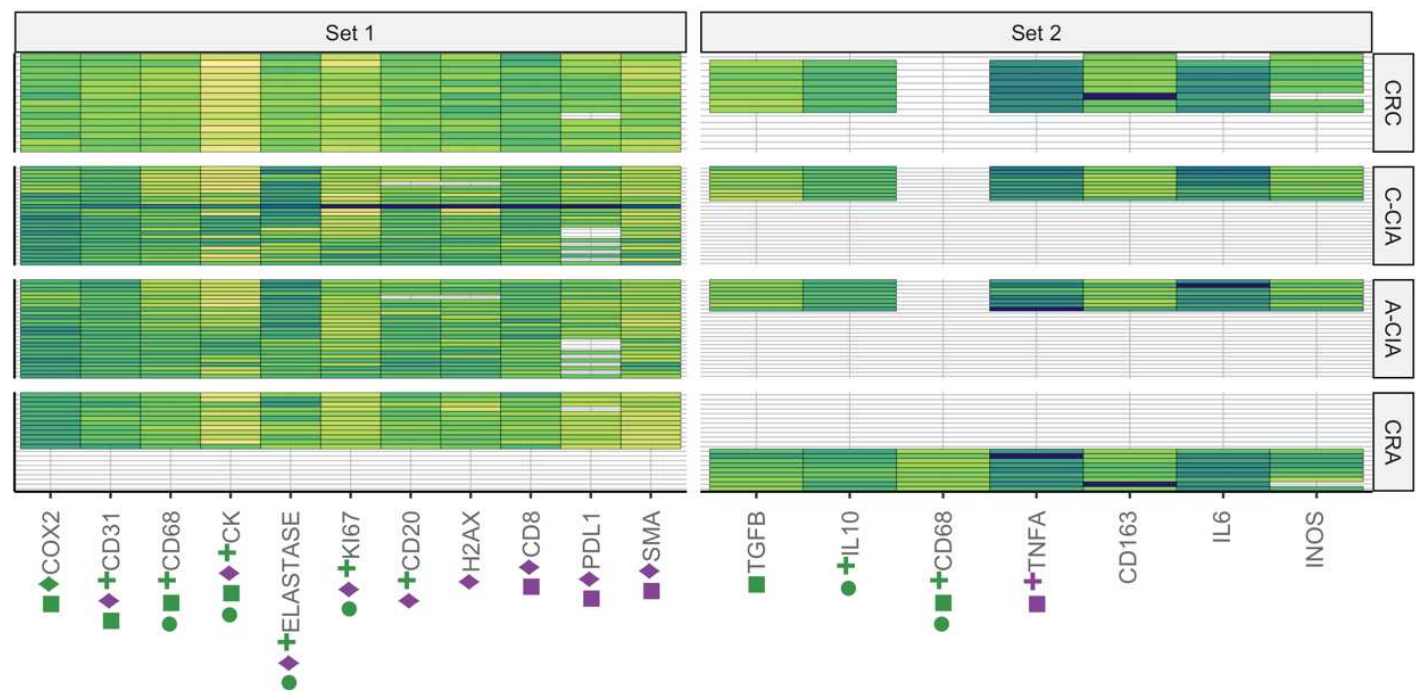

A-CIA to C-CIA to CRC

- Paired A-CIA to C-CIA $\diamond$ CRA to A-CIA

CRA to CRC

Figure 5. Significant changes in average cell type abundance and cytokine expression profiles show that immunosuppressive niche engineering is observed early at the A-CIA stage and continues through CRC. In panels A-C, in addition to showing the distribution of abundances, we also provide histological examples of differences in abundance/gene expression in each category. A) Benign adenomas (CRA) have significantly higher cytotoxic T-cell abundances than progressed adenomas (A-CIA) or carcinomas (CRC). See Figure S7 for $p$-values. B) Changes that accompany the transition from adenoma to carcinoma were revealed using paired tests to directly compare adenomas (A-CIA) to their descendant carcinomas (C-CIA). Here, we show the significant increase in neutrophils from each A-CIA to its descendant C-CIA. C) Expression of the inflammatory cytokine TNF- $\alpha$ (red) significantly decreased during progression from progressed adenoma (A-CIA) to early carcinoma (C-CIA) to malignancy (CRC), culminating in CRC having significantly less TNF- $\alpha$, and more immunosuppressive TGF- $\beta$ (blue) than benign adenomas (CRA). D) Table showing the abundance of each marker (columns) for each sample (rows). Shapes below each marker signify that there were significant changes in abundances across the specified group. Green indicates that the marker increased from the first group to the last group, while purple indicates that the abundances decreased. Examination of changes in cell and cytokine abundances reveal that, among other things, there is a significant decrease in the abundance of cytotoxic T-cells from benign adenoma (CRA) to adenomas that progressed (A$\mathrm{CIA}$ ) (purple diamond). Paired tests reveal that when directly compared to their ancestral adenoma (A-CIA), carcinomas (C-CIA) have significant increases in the abundance of macrophages (CD68), neutrophils (elastase), and cytokine IL-10, all of which can be associated with immune suppression. These trends are also observed when quantifying changes from late adenoma (A-CIA) to nascent carcinoma (C-CIA) to mature carcinoma (CRC), along with additional increases in B-cells (CD20) and vasculature (CD31), and accompanied by a decrease in the inflammatory cytokine TNF- $\alpha$. Together, these patterns indicate that during the evolution of a malignant tumor, engineering of an immunosuppressive ecology begins early in the adenoma stage, and continues through to malignant $\mathrm{CRC}$ formation. 
Our modeling predicts that early acquisition of immune suppression is the dominant escape strategy in CRC, and is crucial for progression. Given these expectations, we selected a panel of 17 markers to describe the immune response in each tumor type (Figure 4 and Supplemental Figures S2-S3). Samples were stained in two sets: the first set comprised $12 \mathrm{CRA}, 25 \mathrm{CIA}$, and $15 \mathrm{CRC}$, with IHC staining for tumor cells (CK), cytotoxic T-cells (CD8), neutrophils (elastase), macrophages (CD68), B-cells (CD20), immune checkpoint inhibition (PD-L1), vasculature (CD31), proliferation (Ki67), fibroblasts (aSMA), DNA damage $(\mathrm{yH} 2 \mathrm{AX})$, and COX2. The second set of samples comprised $9 \mathrm{CRA}, 9 \mathrm{CIA}$, and $9 \mathrm{CRC}$, with IHC staining for macrophages (CD68), M1 macrophages (iNOS) and M2 macrophages (CD163), and RNA in situ hybridization (ISH) for expression of immunosuppressive cytokines IL-10 and TGF- $\beta$, and inflammatory cytokines TNF- $\alpha$ and IL-6. Fifteen cases were in both sets and therefore were stained with all 17 markers. These cases are referred to as the "Intersection Set".

We then examined how cell-type abundances, and the tumor and microenvironment as a whole, changed through progression. The data was generated by creating whole-slide composite images constructed from registered serial slices stained using multi-color IHC and/or RNA ISH (Figures 4, S2, S3). This composite image was then divided into $250 \mu \mathrm{m} \times 250 \mu \mathrm{m}$ quadrats, and the number of pixels positive for each stain was determined for each quadrat (Figures 4 and S4-S6). Quadrat counts were used to perform a spatial analysis, while the abundance of each cell-type was approximated by dividing the total number of positive pixels by the total area sampled. See Supplemental Figures S7 and S10 for details of the statistical tests and significance values. 

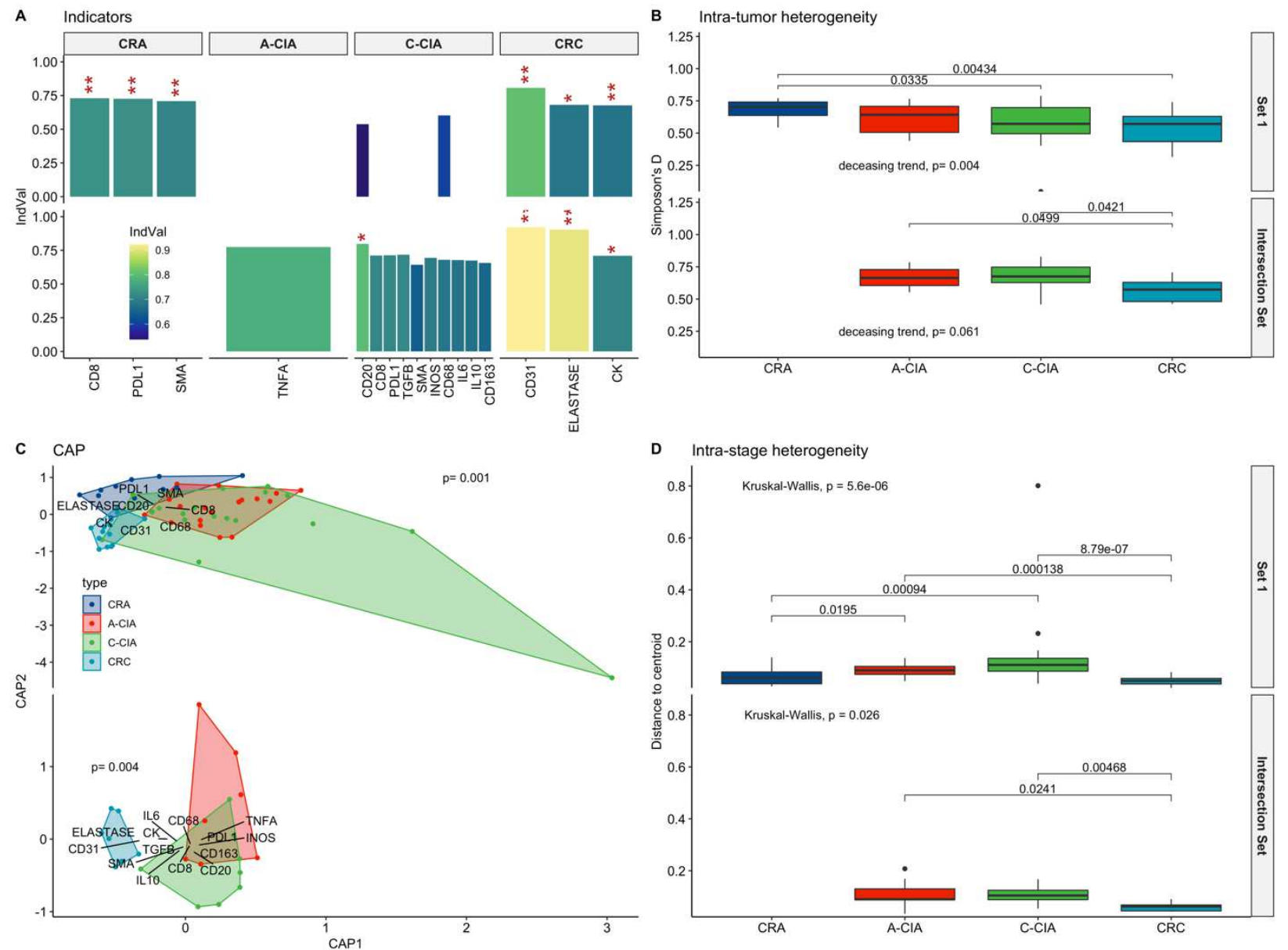

Figure 6. Whole-tissue ecological analysis reveals adenomas and carcinomas have unique and distinct hot and cold ecologies, respectively. A) Indicator species analysis was performed to determine which, if any, cells or cytokines define each tumor stage. The indicator value (IndVal), describes how strongly each cell/cytokine defines the tumor stage. CRA are uniquely defined by cytotoxic Tcells (CD8), PD-L1, and fibroblasts (SMA), while CRC are defined by tumor cells (CK), vasculature (CD31), and neutrophils (elastase). B) Intra-tumor heterogeneity, as measured by Simpson's index. Intra-tumor heterogeneity decreases, as might expected if immune suppression reduces the abundance of pro-inflammatory cells (Jonckheere-Terpstra test for decreasing trends) C) Plotting results from constrained analysis of principal coordinates (CAP) of ecological dissimilarities reveals that CRA and CRC have unique mixes of cell types that create significantly distinct ecologies, as determined by PERMANOVA tests. D) CRC ecologies are significantly more similar to one another than the precursor $\mathrm{A}-\mathrm{CIA}$ and $\mathrm{C}-\mathrm{ClA}$, suggesting $\mathrm{CRC}$ have converged to a common immune-cold ecology defined by vasculature, tumor cells, and neutrophils. This was determined using PERMDISP2, which describes the amount of intrastage ecological heterogeneity by comparing intra-group dispersions of ecological dissimilarities. In the boxplots in panels $B$ and $D$, the center line indicates the median, the top and bottom of the box indicate the $75^{\text {th }}$ and $25^{\text {th }}$ percentiles, respectively, the top whisker the largest value that is no further than 1.5 Interquartile range (IQR) from the $75^{\text {th }}$ percentile, the bottom whisker the smallest value no more than $1.5 \mathrm{IQR}$ from the $25^{\text {th }}$ percentile, and points indicate outliers. Pairwise significance of differences was determined with Dunn's test of multiple comparisons using rank sums.

Our modelling predicted an increase in immunosuppressive cells and a drop in anti-tumor inflammation during the evolution from A-CIA to C-CIA to CRC. Correspondingly, the immunosuppressive cytokine IL-10 showed a significant increase in expression through progression, and this correlated with greater numbers of putatively tumor-promoting neutrophils (elastase) and macrophages (CD68) (Figure 5). Anti-tumor cytotoxic CD8 T cells were progressively excluded through progression (Figure 5A). There was also a significant drop in TNF-a through progression, a powerful pro-inflammatory cytokine (Sethi, Sung, \& Aggarwal, 2008). These patterns were directly evident within individual CIA lesions (e.g. comparing A-CIA directly to its descendant $\mathrm{C}-\mathrm{ClA}$ ). These changes culminate in an immunosuppressed, pro-growth ecology that supports a higher abundance of tumor cells (CK) in CRC, having significantly less inflammation (TNF$\alpha$ and CD8) and more pro-tumor factors, including macrophages (CD68), TGF- $\beta$, and vasculature (CD31) as compared to benign adenomas (CRA). Together, these data suggest that CRA elicit a strong anti-tumor 
immune response, and the evolution of an immunosuppressive microenvironment is associated with progression to CRC.

We next used ecological methods to describe and compare the overall cellular compositions of tumors as a whole, as opposed to one cell type/cytokine at a time. Indicator species analysis (ISA) determines which cell types (or factors), if any, uniquely define a group of tumors (Caceres, 2013). First, we explored the cell types that defined tumor stages. ISA revealed that a defining characteristic of benign CRA is an abundance of CD8+ T cells and PD-L1 expression, consistent with the notion that CRA remain under immune attack but avoid elimination due to having a protective mechanism (Figure 6A). Indicator species characteristic of CRC were an abundance of tumor cells, increased vasculature, and potentially-immunosuppressive neutrophils. Alternative analysis using constrained principal coordinates (CAP) and permutational multivariate analysis of variance (PERMANOVA) (Marti J. Anderson, 2001; Marti J. Anderson \& Willis, 2003; Oksanen et al., 2018) (Figure 6C) confirmed these results. This further reiterates that CRA are defined by T-cell inflammation, while CRC are defined by immune suppression.

If tumors are engineering an immunosuppressive niche to escape immune predation, as predicted by the model, we would expect to see a correlation between differences in tumor microenvironment and differences in immune composition. We tested for this relationship using the Mantel test (Legendre \& Legendre, 2012) to detect correlations between the ecological dissimilarities in immune-cell lineages ( $T$ cells, B cells, macrophages and neutrophils) and dissimilarities in non-immune cells of the microenvironment (tumor cells, fibroblasts, PD-L1, and vasculature) that could affect the behavior of the aforementioned immune cells. Results indicate that differences in immune composition correlate significantly with differences in non-immune ecology $(p=0.003)$, indicating there is a significant relationship between environmental similarity and immunological similarity (Figure S8).

Our initial modeling (Figure 1) predicted that immune suppression is under strong positive selection, as it offers a significant advantage over other escape strategies. This selective process would be expected to decrease both intra-tumor and intra-stage heterogeneity, as we would expect a shift from a mixed immune response (pro- and anti- inflammatory cells) to one dominated by immunosuppressive cells (lower intratumor heterogeneity), resulting in a cold immune ecology common to carcinomas (low intra-stage heterogeneity). To test these predictions, we measured intra-tumor heterogeneity using Simpson's index and compared across stages, finding a significant drop in ecological diversity from CRA to CRC $(p=0.0052$, Jonckheere-Terpstra test for decreasing trends) (Figure 6B). Intra-stage heterogeneity was calculated using PERMDISP2 (M. J. Anderson, 2006), which tests for significant differences in intra-group homogeneity across groups, where here groups are tumor stages. This analysis revealed that CRCs are more homogeneous than precursor A-CIA and C-CIA (Figure 6D). That is, CRC have ecologies more similar to one another, than A-CIA are to one another or C-CIA are to one another. We believe this is because many microenvironmental changes would occur during tumor evolution and construction of the immunosuppressive niche, and the CIA samples (A-CIA, C-CIA) are at different points along this trajectory, and so exhibit higher intra-stage heterogeneity. However, all of the microenvironmental changes eventually lead to the cold immune ecology shared by CRC, hence a lower intra-stage heterogeneity found in the final CRC stage.

\section{Carcinomas are isolated from immune attack}

We next determined how spatial associations between cell types and cytokines change during tumor progression (Figure 7C-F, S9-10). We created 'species association networks' (Popovic, Warton, Thomson, Hui, \& Moles, 2019) that quantify the spatial co-localization between cell types using the data derived from quadrat counts (Figure 7). This method has an advantage over some other spatial statistics, such as correlation or nearest-neighbor distances, as each network describes the conditional dependence relationships between all species, rather than only pairwise spatial distances or correlations in isolation. By accounting for the spatial distribution of all cell types at once, these networks are able to remove indirect effects (i.e. cases where species $A$ and $B$ are correlated because they both interact with $C$ but do not interact with each other) that can confound some other spatial statistics. By controlling for these indirect effects, we have more confidence that the spatial associations are valid. The result is a description of how species are associated in space, after controlling for all other species in the dataset. The resulting complex 
collection of individual pairwise spatial associations (Figure S9) were then organized into meaningful cell type/cytokine spatial clusters by performing community detection on each stage's averaged network (Figure 7C-F). This was accomplished using the Leiden algorithm to detect communities on each tumor stage's multiplex graph (one graph of average positive associations and second graph of average negative associations) (Mucha, Richardson, Macon, Porter, \& Onnela, 2010; Traag, Waltman, \& van Eck, 2019).
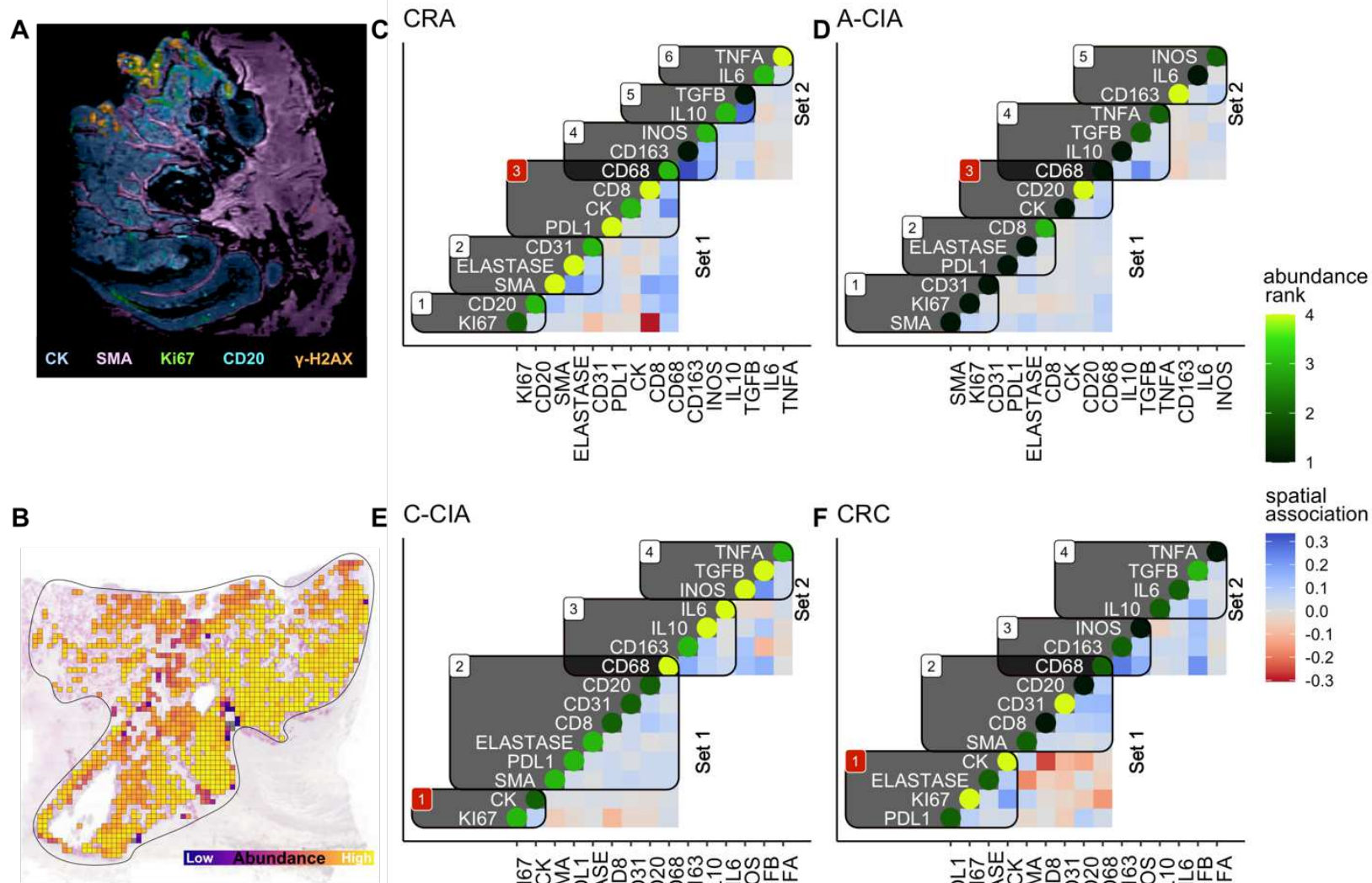

E C-CIA
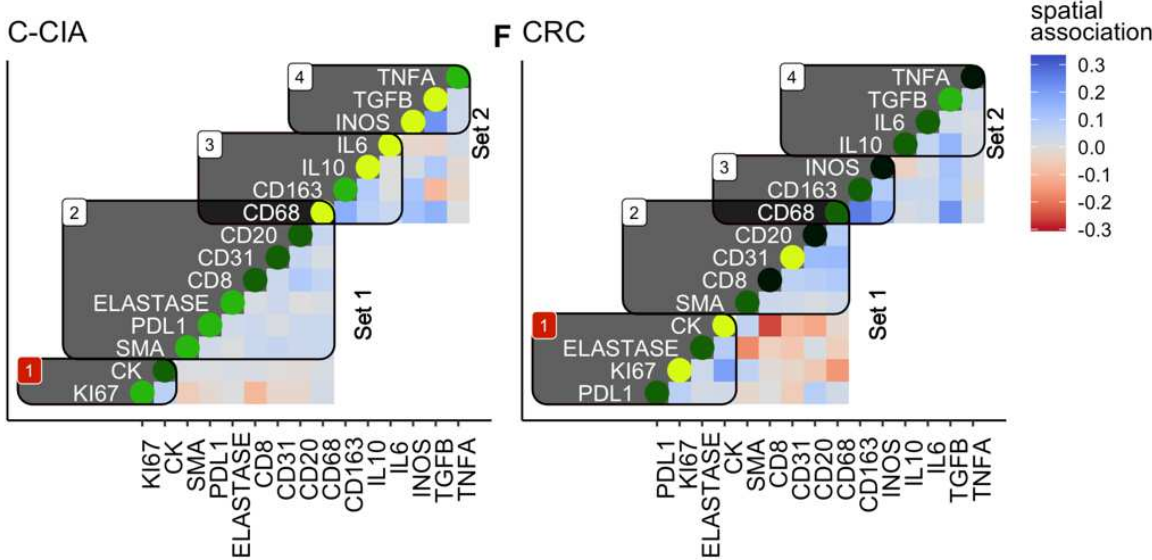

Figure 7. Changes in direct spatial associations. A) Composite images were created by aligning each sample's collection of serial slices. This example shows only 5 markers, but all markers were used for the spatial analysis. B) Each composite image was then divided into quadrats 250 microns in width and height, wherein each marker was quantified at $40 x$ magnification. This image shows the quadrat positions overlaid on one image in the aligned series. C-F) Averaged spatial association networks, clustered by spatial communities (black boxes), with the tumor community labeled in red. The order of cell types within each community does not have meaning. The color of each element in the lower diagonal indicates the average spatial association between the marker pair, with positive blue values indicating clustering, and negative red values meaning cell types/cytokines are found in separated areas. The color of the circle on the diagonal denotes the ranked abundance, e.g. CD8 is highest in CRA (yellow circle) and lowest in CRC (black circle). The interaction networks for both sets have been overlaid on one another using CD68, the only marker found in both datasets (Set 1 and Set 2). Tumor cells in CRA cluster with a mix of pro- and anti-tumor immune cells, suggesting that an anti-tumor immune response is being mounted despite presence of immune escape mechanisms. In contrast, tumor cells in progressed adenomas (A$\mathrm{CIA}$ ) reside in a predominantly immunosuppressive niche, being associated with B-cells (which can promote an M2 macrophage phenotype) and macrophages, which are in turn clustered with immunosuppressive cytokines (IL-10, TGF- $\beta$ ). 
Benign adenomas (CRA) showed signs of being locked in a battle between pro- versus anti-inflammatory cells, as tumor cells clustered with cytotoxic T cells and macrophages, which in turn clustered with both anti-tumor M1 (iNOS) and pro-tumor M2 (CD163) macrophage markers (cluster 3-4 in Figure 7C). It is worth noting that macrophages are plastic and can express both $\mathrm{M} 1$ and $\mathrm{M} 2$ associated markers, so the observation that the CRA macrophages express both markers suggests that they were not polarized to the extremes of either the M1 or M2 phenotypes (P. J. Murray et al., 2014). CRA was the only stage where tumor clustered with cytotoxic T cells, and also had significantly higher abundances of cytotoxic $T$ cells (compared to A-CIA and CRC), and inflammatory TNF- $\alpha$ (compared to CRC) (Figure 5). Together, these observations suggest that the immune system is successfully mounting an attack against tumor cells (cytotoxic T-cells, M1 macrophages), despite tumor cell PD-L1 expression and presence of M2 macrophages (CD68 and CD163).

In contrast to benign adenomas (CRA), the spatial organization of progressed adenomas (A-CIA) was broadly distinct, nevertheless with some similar individual pairwise spatial associations (Figures S9-10). Instead of clustering with cytotoxic T cells, tumor cells in A-CIA cluster with macrophages (CD68) and Bcells (CD20), both of which can produce IL-10 (also found in the A-CIA tumor cluster). IL-10 is known to polarize macrophages towards the M2 phenotype (cluster 3 in Figure 7D) (Affara et al., 2014; Andreu et al., 2010; A. Mantovani et al., 2013; Wong et al., 2010). The macrophages in A-ClA also cluster with the immunosuppressive cytokine TGF- $\beta$, which can too polarize macrophages towards the M2 phenotype. These observations strongly suggest these macrophages are of the immunosuppressive M2 type (cluster 4) (A. Mantovani et al., 2013). With the exception of TNF- $\alpha$, clusters $3 \& 4$ suggest that tumor cells in progressed adenomas exist within a predominantly immunosuppressive niche, with low CD8 T-cell infiltration (note the negative spatial association between CD8 and CK).

Tumor cells in nascent carcinomas (C-CIA) appear to be highly proliferative, clustering with Ki67 (cluster 1, Figure 7E), while having weak spatial associations with immune cells (clusters 2), a trend that continues in CRC. Compared to benign adenomas, tumor cells in CRC having significantly weaker spatial associations with cytotoxic T cells (Figure S9), and significantly lower abundances of cytotoxic T cells and TNF- $\alpha$ (Figure 5 ), suggesting that the anti-tumor response has been suppressed in CRC, which frees tumor cells to divide at higher rates (increased CK/Ki67 spatial association).

\section{Benign adenomas are highly immunogenic, while immune cold carcinomas exhibit antigenic neutrality}

As neoantigens elicit an immune response, it should be expected that changes in the tumor ecology that alter the immune composition will lead to changes in alTH. Our modelling suggests that under immune predation the average neoantigen recognition potential should be low, as tumors must rely on the "Get Lucky" strategy. However, immune escape reduces the selection pressure against high antigenicity, permitting the existence of clones bearing neoantigens that have high recognition potentials (Figures $1 \mathrm{~A}$ and 3D). With the relaxation of selection pressures, antigenicity effectively becomes a quasi-neutral trait, resulting in an increase in alTH and neoantigen burden. Given this understanding of the relationship between the tumor ecology and alTH, we next used multi-region neoantigen prediction from whole exome sequencing (WES) to qualitatively infer the selection pressures experienced during each stage of progression. 

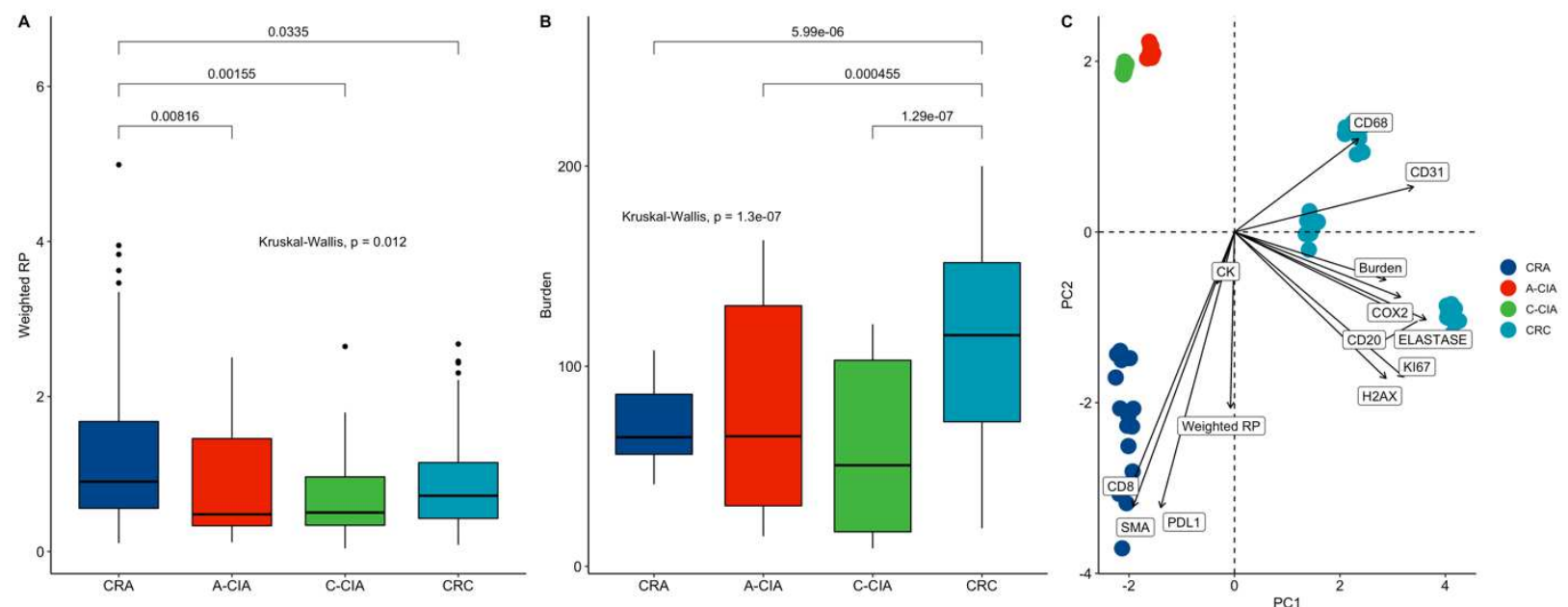

Figure 8. Observed patterns of alTH indicate benign CRA are highly antigenic and remain under immune control, while CRC have escaped elimination via immune suppression. A) Distribution of neoantigen recognition potentials (RP), weighted by their variant allele frequency (VAF). Comparisons across groups indicate that currently benign CRA are significantly more antigenic than all other tumor types, as determined using Dunn's test of multiple comparisons using rank sums. B) CRC have significantly higher neoantigen burdens than all other cell types, and modelling indicates that this occurs when selection against antigenicity is relaxed due to immune suppression. C) Principal component analysis (PCA) of all cell/environmental markers, neoantigen burden, and weighed recognition potentials reveals that CRA are highly antigenic and inflammatory, while CRC are associated with immunosuppressive cells and high neoantigen burden ( $n=2$ CRA, 1 CIA, 3 CRA).

CRA were the most immunogenic of all samples, with significantly higher mean neoantigen recognition potentials, when weighting each recognition potential by its associated neoantigen's variant allele frequency (VAF) (Figure 8A). Conversely, CRC had the highest number of predicted neoantigens of any tumor stage (Figure 8B), but fewer neoantigens at high VAF which also had high recognition potential. These observations are consistent with our modelling predictions that neoantigens are free to accumulate following escape from immune predation, and our ecological measurements indicating the establishment of a T-cellexcluded immunosuppressive microenvironment in CRC, but not adenomas.

We used principle components analysis (PCA) to gain a holistic picture of the tumor-immune ecoevolutionary dynamics by examining alTH, tumor ecology, and tumor stage together (analysis restricted to samples for which we had both genomic and histological data; Figure $8 \mathrm{C}$ ). The first two components, which explained $67.3 \%$ of the variance ( 44.5 by PC 1 , and $22.8 \%$ by PC2), were positively correlated, with negative PC1 and PC2 associated with inflammation (cytotoxic T cells, weighted recognition potentials). Larger PC1 and PC2 values were associated with growth and immune suppression (proliferation, vasculature, macrophages, neutrophils) and an increased neoantigen burden that would be expected to accompany a loss of selection against antigenicity, much like how neutral mutations accumulate freely (Lakatos et al., 2019). Benign CRA and malignant CRC samples prove to be opposites, with CRA clustering in the inflammatory "corner", and CRC clustering in the opposite immunosuppression, pro-growth corner.

Model predicts benign adenomas are unable to overcome immune attack despite signs of immune suppression. 
A

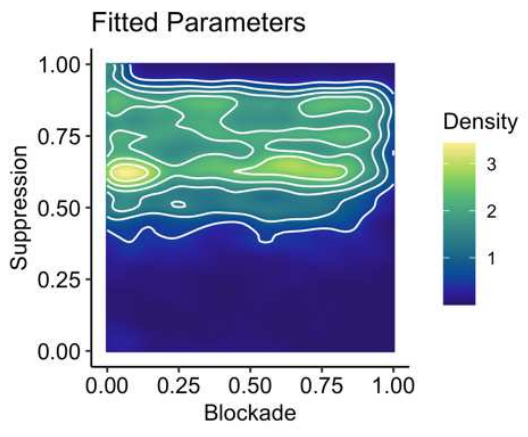

D

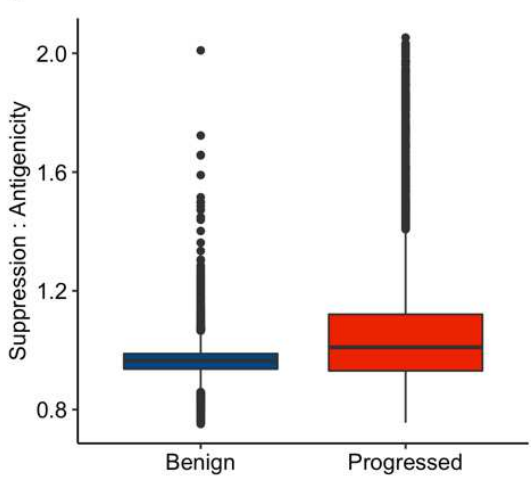

B

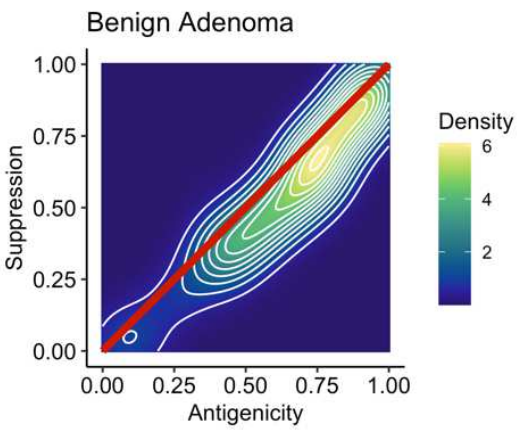

E

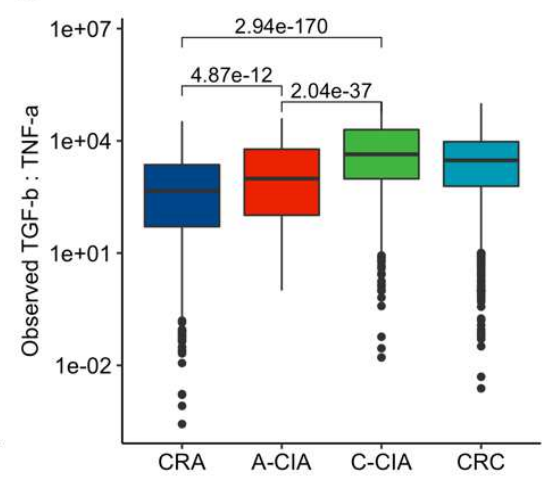

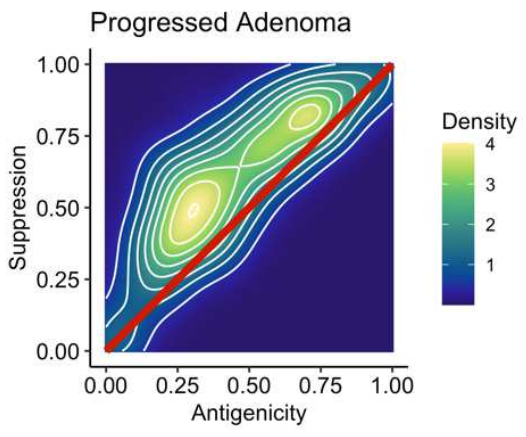

F

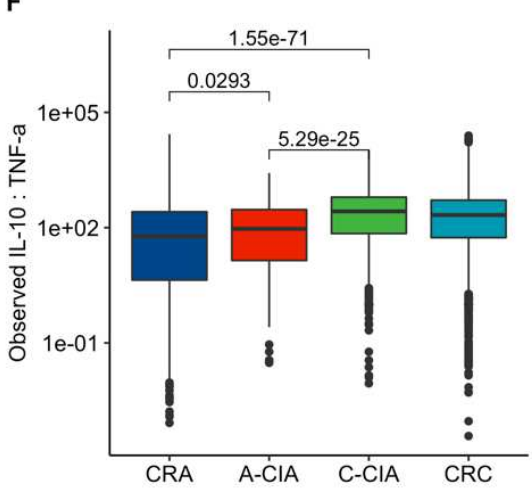

Figure 9. An accept/reject statistical inference method was used to determine which model parameters could recreate the observation that benign CRA were more immunogenic and also had more immunosuppressive cells than progressed adenomas (A-CIA). A) Heatmap showing the number of times each combination of Blockade $(\phi)$ and Suppression $(\sigma)$ produced a benign CRA that had higher antigenicity, more immune suppression, and more immune blockade than progressed adenomas (A-CIA) using that same parameter combination. The narrow range of high suppression values $(0.583-0.83,25$ th and 75 th percentile, respectively), and wide range of immune blockade values (0.167-0.71, 25th and 75th percentile respectively), suggests that, in colorectal cancer, immune suppression is strong, and the effect of blockade is minimal, as there is no clear gradient along the Blockade axis. B, C) Heatmaps showing the relationship between antigenicity and immune suppression of simulated benign and progressed adenomas that fit the data, with the red line showing a 1:1 relationship. For any given antigenicity, simulated progressed adenomas have more immune suppression compared to benign adenomas D) Ratio of suppression to antigenicity in simulated benign and progressed adenomas. Progressed adenomas' higher ratio allows them more effectively reduce immune predation. E, F) Observed spatial ratios of immunosuppressive cytokines (TGF- $\beta$, IL-10) to inflammatory cytokines (TNF- $\alpha$ ), based on marker abundances within each samples' quadrats. As predicted by modeling, compared to benign adenomas (CRA), progressed adenomas (A-CIA) have significantly higher ratios of immunosuppressive cytokines to inflammatory cytokines. While the effects of cytokines may not be equal, these results suggest that effect of immune suppression will be greater in progressed adenomas than in benign adenomas.

A surprising finding of the ecological analysis was that progressed (A-CIA) and benign adenomas (CRA) exhibit similar levels of some immunosuppressive markers, such as similar abundances of macrophages (CD68), M2 markers (CD163), and immunosuppressive cytokines (IL-10, TGF- $\beta$ ), with CRA even having significantly stronger positive spatial associations between CD163/CD68 (M2 macrophages) (Figure 5, S910). However, this appears to be counterbalanced by an anti-tumor inflammatory response, with CRA (compared to A-CIA) having significantly higher levels of CD8 and weighted neoantigen recognition potentials, whilst also forming spatial clusters consisting of a mix of pro- and anti-inflammatory cells (Figures $5-8)$.

Both progressed and benign adenomas (A-CIA and CRA, respectively) show signs of immune suppression. We hypothesized that the key enabler of progression to invasive disease is the development of more effective immune suppression by eventually-invasive lesions that is sufficient to overcome the higher immunogenicity of the progressor lesions. To test the plausibility of this hypothesis, we returned to our model to compare benign and progressed adenomas in simulations where benign adenomas had similar/higher levels of immune suppression and greater inflammation compared to progressed adenomas. 
We used a Monte Carlo accept-reject method to find model parameters that reproduced our observations that, compared to progressed adenomas (A-CIA), benign adenomas (CRA) were: significantly more immunogenic (higher CD8 abundances, higher neoantigen recognition potentials, residing in spatial niche containing CD8 cells and M1 macrophages); had more immune blockade (PD-L1); similar levels of immunosuppressive cells/cytokines (CD68, CD163, IL-10, TGF- $\beta$ ) (data analyses in Figures 5-8). (Figures 9, S11).

The parameters that reproduced our observations all had a narrow range of high immune suppression values, $0.58-0.83\left(25^{\text {th }}\right.$ and $75^{\text {th }}$ percentile), but a wide range of immune blockade values $0.17-0.71$ ( $25^{\text {th }}$ and $75^{\text {th }}$ percentile) (Figure 9A). Within these simulations, the difference between adenomas that progressed and those that remained benign was that the former had a higher ratio of immune suppression to antigenicity (Figure 9B-D). Going back to the data, we then quantified the ratio of immunosuppressive cytokines (TGF- $\beta$, IL-10) to inflammatory cytokines (TNF- $\alpha$ ) in benign and progressed adenomas (Figure $9 \mathrm{E}-\mathrm{F})$. In both cases, we found that progressed adenomas had significantly higher ratios of immunosuppressive cytokines to inflammatory cytokines. These findings are consistent with the model prediction that a key difference between progressed and benign adenomas is that immune suppression is more effective in progressed adenomas due to them having a reduced inflammatory response, making it easier to overcome. This builds upon the model's general conclusion that immune suppression is expected to be present at tumorigenesis, adding that immune suppression must also be sufficiently strong to overcome the tumor's immunogenicity, which is most easily accomplished by having immune suppression coupled with low immunogenicity: the pattern observed in progressed adenomas (A-CIA).

Additionally, model fitting also suggests that immune suppression is fairly strong in colorectal cancer, as most fitted values had high levels of immune suppression (Figure 9A). It seems also that the role of PD-L1 is minimal, as there was a wide range of immune blockade strengths that fit the data. In other words, high protection from blockade occurred as frequently as low protection, likely because it is playing a secondary, almost supportive, role to immune suppression, and therefore the added benefit is minimal.

\section{Discussion}
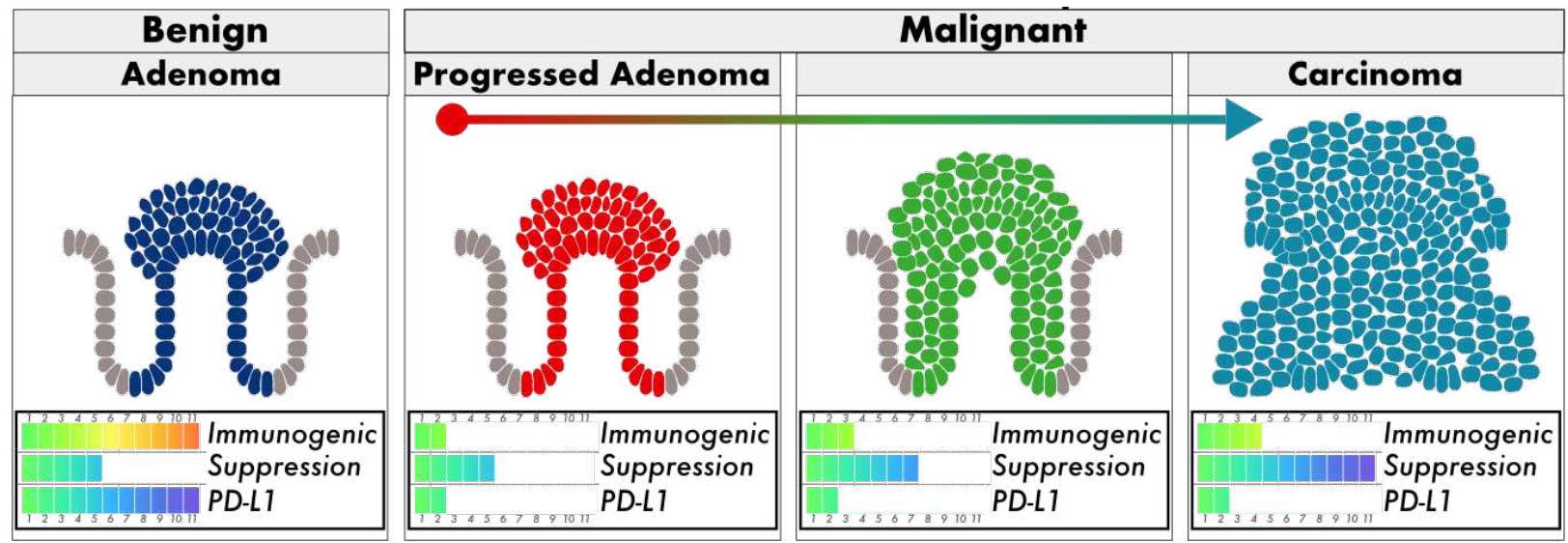

Figure 10. Modelling, imaging, and genomic analyses indicate that benign CRA are highly antigenic (i.e. 'Unlucky') and remain under immune control, despite having some immune suppression. This suggests that benign CRA are stuck at the immunogenic bottleneck. CRA that do progress instead appear to initially survive using the Get Lucky strategy, while eventually shifting to a Get Smart one as immune suppression strengthens. As immune escape relaxes selection against antigenicity, CRC rely less on Get Lucky, and are able to accumulate neoantigens, increasing neoantigen burden. These conclusions are consistent with the observation that antigenicity and cytotoxic T-cells are good prognostic markers, but also suggest that altering the immunosuppressive ecology, as opposed to targeting checkpoint inhibitors, may prove to be a more successful treatment strategy.

Integration of mathematical modeling, ecological analysis of whole slide images, and quantification of intratumor antigenic heterogeneity (alTH) paints a clear picture of the origins and progression of human colorectal cancer. Benign adenomas are unable to pass through an immunogenic bottleneck due to high immunogenicity and insufficient immune suppression. Conversely, adenomas that progress do so by 
avoiding anti-tumor inflammation via immune suppression that is sufficiently strong to overcome their significantly lower immunogenicity. The immunosuppressive niche of progressed adenomas continues to expand during progression to carcinoma, resulting in a highly proliferative tumor existing within an immunecold ecology.

This conclusion is supported by the observations that, compared to progressed adenomas $(A-C I A)$, benign adenomas (CRA) exhibit significantly higher abundances of cytotoxic T cells, likely due to their also significantly higher immunogenicity (Figures 5D and 8A). These higher levels of cytotoxic T cells and immunogenicity are so severe that they drive unique ecological and genomic signatures for CRA (Figures $6 \mathrm{~A}$ and $8 \mathrm{C}$ ). CRA also exist within a spatial community of both pro- and anti-tumor immune cells (Figure $7 \mathrm{C}$ ), suggesting that the immune system is successfully mounting an attack against the tumor, despite the presence of immunosuppressive cells and cytokines. In contrast, progressed adenomas reside within a predominately immunosuppressive spatial community (niche), separated from cytotoxic T-cells (Figure 7D). While progressed adenomas (A-CIA) have similar abundances of immunosuppressive cells and cytokines, another critical difference is that A-CIA have significantly fewer cytotoxic T-cells and lower antigenicity (Figures 5D, 8A). Together, these observations suggest that, unlike benign adenomas (CRA), the combination of lower immunogenicity and an immunosuppressive niche more successfully reduces immune predation, which will allow the tumor to persist and eventually progress. Throughout progression from adenoma to carcinoma (A-CIA to C-CIA to CRC), there is a trend of decreasing abundances of cytotoxic T cells and TNF- $\alpha$, coupled with increases of tumor, M2 macrophage markers, and immunosuppressive cytokines (Figure 5D), suggesting that immune suppression continues to strengthen. The net result of these changes is the generation of a homogenous immune-cold ecology common to carcinomas, allowing them to reside within a spatial community isolated from the immune system, where they are free to divide and accumulate neoantigens (Figures 6B, 6D, 7F, 8A-C).

Our data-driven hypothesis that CRA remain controlled due to an anti-tumor immune response that is minimally suppressed is consistent with our mathematical model, where we showed that benign adenomas have a lower ratio of immune suppression to antigenicity, compared to adenomas that progressed (Figure 9B). While it has been shown that inflammation is a marker of good prognosis (Galon et al., 2006; Pages et al., 2005; Pages et al., 2009), and immune suppression is a marker of poor prognosis (Hanahan \& Weinberg, 2011; Pinto et al., 2019; Steidl et al., 2010), our hypothesis suggests that it is important to consider both inflammation and immune suppression when assessing the risk of progression. If we had considered only the abundance of immunosuppressive cells and cytokines, we might have predicted that our benign adenomas would progress. However, when we incorporated tumor immunogenicity and the spatial organization of the tumor microenvironment, we saw clear signs that the immune system was mounting a response against the tumor, despite the presence of immunosuppressive cells and cytokines. In other words, without considering both inflammation and immune suppression, we might have mistakenly predicted the adenoma had a high risk of progression.

A second clinical implication of our work is that immune blockade appears to play little role in the pathway to malignancy. Modeling suggests that immune suppression is the superior strategy, and that it will outcompete those relying solely on blockade (Figures 1 and 3 ). This is because, similar to immune blockade, immune suppression offers protection, but also creates an environment more supportive of tumor growth. These features of immune suppression make blockade redundant: if there is already immune suppression, blockade offers little to no additional benefit. Our ecological analysis reinforces this prediction that immune suppression obviates the need for immune blockade, for example when compared to benign adenomas, PD-L1 is found at significantly lower levels in progressed adenomas and carcinomas, suggesting it does not play an important role in tumorigenesis or progression (Figure 5D). Thus, we suspect that while PD-L1 may help prevent CRA from being eliminated at the very earliest stages, it is not an important feature of any stage of the pathway from A-CIA to C-CIA to CRC.

Our hypothesis that PD-L1 does not play a large role in tumorigenesis or progression may help explain why MSI-low tumors respond poorly to immune checkpoint inhibitors (Ganesh et al., 2019; Kalyan, Kircher, Shah, Mulcahy, \& Benson, 2018; Passardi, Canale, Valgiusti, \& Ulivi, 2017). Similarly, given that immune suppression appears to be the dominant escape strategy, and that CD8 T cells are isolated from the tumor, we predict that therapies designed to increase cytotoxic T-cell killing, such as dendritic cell vaccines and chimeric antigen receptor (CAR) T-cell therapy, would have limited success unless the immunosuppressive niche is addressed with additional therapy. 
Given that the immunosuppressive niche seems to be the driver of immune escape, more effective treatments of colorectal cancer might be those that seek to re-engineer a hot immune ecology, possibly by re-polarizing immunosuppressive cells, a treatment currently being explored for macrophages (Brown, Recht, \& Strober, 2017; Genard, Lucas, \& Michiels, 2017; Kulkarni et al., 2018; Myers, Pienta, \& Amend, 2020). An additional benefit to re-engineering the immunosuppressive niche is that doing so could shift the fitness landscape for all cells within the tumor. This is in contrast to targeted therapies aimed at eliminating only those cells that carry a particular mutation, an approach that suffers from almost inevitable acquired resistance and relapse. Thus, a major benefit to re-engineering the immunosuppressive niche is that by targeting a common underlying mechanism of tumorigenesis, it casts a wider net on the heterogeneous population of cells, potentially reducing the risk of evolving resistance.

In summary, we provide evidence of a critical role for immune predation in preventing colorectal malignancy, implying that immune evasion represents a key bottleneck in disease progression. In colorectal cancer, our analysis strongly suggests that it is the construction of an immunosuppressive niche by the tumor that is the predominant pathway through this bottleneck. As the immunosuppressive niche is fundamental to progression and persistence, re-engineering the microenvironment towards an immune-hot phenotype may prove to be an effective form of immunotherapy. 


\section{Acknowledgements}

CG, MRT and ARAA are supported by Physical Sciences Oncology Network (PSON) grant from the National Cancer Institute (grant no. U54CA193489). MRT, JW and ARAA are also supported the Cancer Systems Biology Consortium grant from the National Cancer Institute (grant no. U01CA23238). ARAA would also like to acknowledge support from the Moffitt Cancer Center of Excellence for Evolutionary Therapy. TG is supported by the Wellcome Trust (202778/Z/16/Z) and Cancer Research UK (A19771). AS is supported by the Wellcome Trust (202778/B/16/Z) and Cancer Research UK (A22909). We acknowledge funding from the National Institute of Health (NCI U54 CA217376) to AS and TAG. This work was also supported a Wellcome Trust award to the Centre for Evolution and Cancer at the Institute of Cancer Research (105104/Z/14/Z). TAG and SL are grateful for support from the Bowel and Cancer Research Charity (project grant scheme). TAG received support from the Barts Charity (pilot grant scheme). SL was supported by Wellcome Trust Senior Clinical Research Fellowship (206314/Z/17/Z).

\section{Data Availability}

Data will be made available on the European Genome-Phenome Archive, including sequencing data and histology images.

\section{Software Availability}

Software will be made available on GitHub.

\section{References}

Affara, N. I., Ruffell, B., Medler, T. R., Gunderson, A. J., Johansson, M., Bornstein, S., ... Coussens, L. M. (2014). B cells regulate macrophage phenotype and response to chemotherapy in squamous carcinomas. Cancer Cell, 25(6), 809-821. doi:10.1016/j.ccr.2014.04.026

Agarwal, M., \& Bhadauria, A. (2013). A generalised prey-predator type model of immunogenic cancer with the effect of immunotherapy. International Journal of Engineering Science and Technology, 5, 66-84. doi:10.4314/ijest.v5i1.6

Alcantarilla, P. F., Bartoli, A., \& Davison, A. J. (2012, 2012//). KAZE Features. Paper presented at the Computer Vision - ECCV 2012, Berlin, Heidelberg.

Anderson, M. J. (2001). A new method for non-parametric multivariate analysis of variance. Austral Ecology, 26(1), 32-46. doi:10.1111/j.1442-9993.2001.01070.pp.x

Anderson, M. J. (2006). Distance-based tests for homogeneity of multivariate dispersions. Biometrics, 62(1), 245-253. doi:10.1111/j.1541-0420.2005.00440.x

Anderson, M. J., \& Willis, T. J. (2003). CANONICAL ANALYSIS OF PRINCIPAL COORDINATES: A USEFUL METHOD OF CONSTRAINED ORDINATION FOR ECOLOGY. Ecology, 84(2), 511-525. doi:doi:10.1890/0012-9658(2003)084[0511:CAOPCA]2.0.C0;2

Andreu, P., Johansson, M., Affara, N. I., Pucci, F., Tan, T., Junankar, S., . . Coussens, L. M. (2010). FcRgamma activation regulates inflammation-associated squamous carcinogenesis. Cancer Cell, 17(2), 121-134. doi:10.1016/j.ccr.2009.12.019

Angelova, M., Charoentong, P., Hackl, H., Fischer, M. L., Snajder, R., Krogsdam, A. M., .. . Trajanoski, Z. (2015). Characterization of the immunophenotypes and antigenomes of colorectal cancers reveals distinct tumor escape mechanisms and novel targets for immunotherapy. Genome Biol, 16, 64. doi:10.1186/s13059-015-0620-6

Babbs, C. F. (2012). Predicting success or failure of immunotherapy for cancer: insights from a clinically applicable mathematical model. Am J Cancer Res, 2(2), 204-213.

Bradski, G. (2000). The OpenCV Library. Dr. Dobb's Journal of Software Tools.

Broad Institute. (2017). Picard Tools: http://broadinstitute.github.io/picard/. 
Brown, J. M., Recht, L., \& Strober, S. (2017). The Promise of Targeting Macrophages in Cancer Therapy. Clin Cancer Res, 23(13), 3241-3250. doi:10.1158/1078-0432.CCR16-3122

Caceres, M. D. (2013). How to use the indicspecies package.

Carulli, A. J., Samuelson, L. C., \& Schnell, S. (2014). Unraveling intestinal stem cell behavior with models of crypt dynamics. Integr Biol (Camb), 6(3), 243-257. doi:10.1039/c3ib40163d

Carvajal-Carmona, L. G., Zauber, A. G., Jones, A. M., Howarth, K., Wang, J., Cheng, T., ... . Tomlinson, I. (2013). Much of the genetic risk of colorectal cancer is likely to be mediated through susceptibility to adenomas. Gastroenterology, 144(1), 53-55. doi:10.1053/j.gastro.2012.09.016

Coffelt, S. B., Wellenstein, M. D., \& de Visser, K. E. (2016). Neutrophils in cancer: neutral no more. Nat Rev Cancer, 16(7), 431-446. doi:10.1038/nrc.2016.52

Coulie, P. G., Van den Eynde, B. J., van der Bruggen, P., \& Boon, T. (2014). Tumour antigens recognized by T lymphocytes: at the core of cancer immunotherapy. Nature Reviews Cancer, 14.

Cross, W., Kovac, M., Mustonen, V., Temko, D., Davis, H., Baker, A. M., ... Tomlinson, I. P. M. (2018). The evolutionary landscape of colorectal tumorigenesis. Nat Ecol Evol, 2(10), 1661-1672. doi:10.1038/s41559-018-0642-z

Cunningham, F., Achuthan, P., Akanni, W., Allen, J., Amode, M. R., Armean, I. M., .. Flicek, P. (2019). Ensembl 2019. Nucleic Acids Res, 47(D1), D745-D751. doi:10.1093/nar/gky1113

De Cáceres, M., \& Legendre, P. (2009). Associations between species and groups of sites: Indices and statistical inference (Vol. 90).

Dinno, A. (2017). conover.test: Conover-Iman Test of Multiple Comparisons Using Rank Sums.

Dunn, G. P., Bruce, A. T., Ikeda, H., Old, L. J., \& Schreiber, R. D. (2002). Cancer immunoediting: from immunosurveillance to tumor escape. Nat Immunol, 3(11), 991-998. doi:10.1038/ni1102-991

Fearon, E. R., \& Vogelstein, B. (1990). A genetic model for colorectal tumorigenesis. Cell, 61(5), 759-767.

Fischler, M. A., \& Bolles, R. C. (1981). Random Sample Consensus: A Paradigm for Model Fitting with Applications to Image Analysis and Automated Cartography. Association for Computing Machinery, 24(6), 381-395.

Galon, J., Costes, A., Sanchez-Cabo, F., Kirilovsky, A., Mlecnik, B., Lagorce-Pages, C., . . Pages, F. (2006). Type, density, and location of immune cells within human colorectal tumors predict clinical outcome. Science, 313(5795), 1960-1964. doi:10.1126/science.1129139

Ganesh, K., Stadler, Z. K., Cercek, A., Mendelsohn, R. B., Shia, J., Segal, N. H., \& Diaz, L. A., Jr. (2019). Immunotherapy in colorectal cancer: rationale, challenges and potential. Nat Rev Gastroenterol Hepatol, 16(6), 361-375. doi:10.1038/s41575-019-0126-x

Gatenby, R. A., \& Vincent, T. L. (2003). Application of quantitative models from population biology and evolutionary game theory to tumor therapeutic strategies. Mol Cancer Ther, 2(9), 919-927.

Gause, G. F., \& Witt, A. A. (1935). Behavior of Mixed Populations and the Problem of Natural Selection. The American Naturalist, 69(725), 596-609. doi:10.1086/280628 
Genard, G., Lucas, S., \& Michiels, C. (2017). Reprogramming of Tumor-Associated Macrophages with Anticancer Therapies: Radiotherapy versus Chemo- and Immunotherapies. Front Immunol, 8, 828. doi:10.3389/fimmu.2017.00828

Giannakis, M., Mu, X. J., Shukla, S. A., Qian, Z. R., Cohen, O., Nishihara, R., . . Garraway, L. A. (2016). Genomic Correlates of Immune-Cell Infiltrates in Colorectal Carcinoma. Cell Rep, 15(4), 857-865. doi:10.1016/j.celrep.2016.03.075

Goode, A., Gilbert, B., Harkes, J., Jukic, D., \& Satyanarayanan, M. (2013). OpenSlide: A vendor-neutral software foundation for digital pathology. J Pathol Inform, 4, 27. doi:10.4103/2153-3539.119005

Grasso, C. S., Giannakis, M., Wells, D. K., Hamada, T., Mu, X. J., Quist, M., . . Peters, U. (2018). Genetic Mechanisms of Immune Evasion in Colorectal Cancer. Cancer Discov, 8(6), 730-749. doi:10.1158/2159-8290.CD-17-1327

Grivennikov, S. I., Greten, F. R., \& Karin, M. (2010). Immunity, inflammation, and cancer. Cell, 140(6), 883-899. doi:10.1016/j.cell.2010.01.025

Hanahan, D., \& Weinberg, R. A. (2011). Hallmarks of cancer: the next generation. Cell, 144(5), 646-674. doi:10.1016/j.cell.2011.02.013

Hofstad, B., Vatn, M. H., Andersen, S. N., Huitfeldt, H. S., Rognum, T., Larsen, S., \& Osnes, M. (1996). Growth of colorectal polyps: redetection and evaluation of unresected polyps for a period of three years. Gut, 39(3), 449-456.

Hothorn, T., Hornik, K., Wiel, M. A. v. d., \& Zeileis, A. (2008). Implementing a Class of Permutation Tests: The coin Package. Journal of Statistical Software, 28(8), 1-23.

Josephidou, M., Lynch, A. G., \& Tavaré, S. (2015). multiSNV: a probabilistic approach for improving detection of somatic point mutations from multiple related tumour samples. Nucleic Acids Research, 43(9), e61--e61. doi:10.1093/nar/gkv135

Jurtz, V., Paul, S., Andreatta, M., Marcatili, P., Peters, B., \& Nielsen, M. (2017). NetMHCpan4.0: Improved Peptide \textendashMHC Class I Interaction Predictions Integrating Eluted Ligand and Peptide Binding Affinity Data. The Journal of Immunology, 199(9), 3360--3368. doi:10.4049/jimmunol.1700893

Kalyan, A., Kircher, S., Shah, H., Mulcahy, M., \& Benson, A. (2018). Updates on immunotherapy for colorectal cancer. Journal of gastrointestinal oncology, 9(1), 160169. doi:10.21037/jgo.2018.01.17

Khan, A. M., Rajpoot, N., Treanor, D., \& Magee, D. (2014). A Nonlinear Mapping Approach to Stain Normalization in Digital Histopathology Images Using Image-Specific Color Deconvolution. IEEE Transactions on Biomedical Engineering, 61(6), 1729-1738. doi:10.1109/TBME.2014.2303294

Khong, H. T., \& Restifo, N. P. (2002). Natural selection of tumor variants in the generation of "tumor escape" phenotypes. Nat Immunol, 3(11), 999-1005. doi:10.1038/ni1102999

Kim, B. G., Li, C., Qiao, W., Mamura, M., Kasprzak, B., Anver, M., ... Letterio, J. J. (2006). Smad4 signalling in T cells is required for suppression of gastrointestinal cancer. Nature, 441(7096), 1015-1019. doi:10.1038/nature04846

King, T., Butcher, S., \& Zalewski, L. (March, 2017). Apocrita - High Performance Computing Cluster for Queen Mary University of London. doi:10.5281/zenodo.438045

Kinzler, K. W., \& Vogelstein, B. (1996). Lessons from hereditary colorectal cancer. Cell, 87(2), 159-170. doi:10.1016/s0092-8674(00)81333-1 
Kulkarni, A., Chandrasekar, V., Natarajan, S. K., Ramesh, A., Pandey, P., Nirgud, J., . . . Sengupta, S. (2018). A designer self-assembled supramolecule amplifies macrophage immune responses against aggressive cancer. Nature Biomedical Engineering, 2(8), 589-599. doi:10.1038/s41551-018-0254-6

Lakatos, E., Williams, M., Schenck, R., Cross, W., Househam, J., Werner, B., ... Graham, T. (2019). Evolutionary dynamics of neoantigens in growing tumours.

Legendre, P., \& Legendre, L. (2012). Numerical ecology (Third English edition. ed.). Amsterdam: Elsevier.

Lek, M., Karczewski, K. J., Minikel, E. V., Samocha, K. E., Banks, E., Fennell, T., ... Exome Aggregation, C. (2016). Analysis of protein-coding genetic variation in 60,706 humans. Nature, 536(7616), 285-291. doi:10.1038/nature19057

Lek, M., Karczewski, K. J., Minikel, E. V., Samocha, K. E., Banks, E., Fennell, T., ... Exome Aggregation, C. (2016). Analysis of protein-coding genetic variation in 60,706 humans. Nature, 536(7616), 285-291. doi:10.1038/nature19057

Li, H., \& Durbin, R. (2010). Fast and accurate long-read alignment with Burrows--Wheeler transform. Bioinformatics, 26(5), 589--595.

Li, H., Handsaker, B., Wysoker, A., Fennell, T., Ruan, J., Homer, N., .. Subgroup, G. P. D. P. (2009). The Sequence Alignment/Map format and SAMtools. Bioinformatics, 25(16), 2078--2079. doi:10.1093/bioinformatics/btp352

Logan, R. F., Patnick, J., Nickerson, C., Coleman, L., Rutter, M. D., von Wagner, C., \& English Bowel Cancer Screening Evaluation, C. (2012). Outcomes of the Bowel Cancer Screening Programme (BCSP) in England after the first 1 million tests. Gut, 61(10), 1439-1446. doi:10.1136/gutjnl-2011-300843

Łuksza, M., Riaz, N., Makarov, V., Balachandran, V. P., Hellmann, M. D., Solovyov, A., ... Greenbaum, B. D. (2017). A neoantigen fitness model predicts tumour response to checkpoint blockade immunotherapy. Nature, 551, 517 EP -.

Mantovani, A. (2014). Macrophages, Neutrophils, and Cancer: A Double Edged Sword. New Journal of Science, 2014, 14.

Mantovani, A., Biswas, S. K., Galdiero, M. R., Sica, A., \& Locati, M. (2013). Macrophage plasticity and polarization in tissue repair and remodelling. J Pathol, 229(2), 176185. doi:10.1002/path.4133

Mascaux, C., Angelova, M., Vasaturo, A., Beane, J., Hijazi, K., Anthoine, G., ... Galon, J. (2019). Immune evasion before tumour invasion in early lung squamous carcinogenesis. Nature.

May, R. (1976). Theoretical ecology : principles and applications. Philadelphia: Saunders.

McGranahan, N., Rosenthal, R., Hiley, C. T., Rowan, A. J., Watkins, T. B. K., Wilson, G. A., . . Consortium, T. R. (2017). Allele-Specific HLA Loss and Immune Escape in Lung Cancer Evolution. Cell, 171(6), 1259-1271 e1211. doi:10.1016/j.cell.2017.10.001

Medema, J. P., de Jong, J., Peltenburg, L. T., Verdegaal, E. M., Gorter, A., Bres, S. A., ... Offringa, R. (2001). Blockade of the granzyme B/perforin pathway through overexpression of the serine protease inhibitor PI-9/SPI-6 constitutes a mechanism for immune escape by tumors. Proceedings of the National Academy of Sciences of the United States of America, 98(20), 11515-11520. doi:10.1073/pnas.201398198

Mucha, P. J., Richardson, T., Macon, K., Porter, M. A., \& Onnela, J. P. (2010). Community structure in time-dependent, multiscale, and multiplex networks. Science, 328(5980), 876-878. doi:10.1126/science.1184819 
Murray, J. D. (2002). Mathematical Biology I. An Introduction (Vol. 17). New York: Springer. Murray, P. J., Allen, J. E., Biswas, S. K., Fisher, E. A., Gilroy, D. W., Goerdt, S., .. Wynn, T. A. (2014). Macrophage activation and polarization: nomenclature and experimental guidelines. Immunity, 41(1), 14-20. doi:10.1016/j.immuni.2014.06.008

Muto, T., Bussey, H. J., \& Morson, B. C. (1975). The evolution of cancer of the colon and rectum. Cancer, 36(6), 2251-2270.

Myers, K. V., Pienta, K. J., \& Amend, S. R. (2020). Cancer Cells and M2 Macrophages: Cooperative Invasive Ecosystem Engineers. Cancer Control, 27(1), 1073274820911058. doi:10.1177/1073274820911058

Ngiow, S. F., von Scheidt, B., Akiba, H., Yagita, H., Teng, M. W., \& Smyth, M. J. (2011). AntiTIM3 antibody promotes T cell IFN-gamma-mediated antitumor immunity and suppresses established tumors. Cancer Res, 71(10), 3540-3551. doi:10.1158/00085472.CAN-11-0096

Oksanen, J., Blanchet, F. G., Friendly, M., Kindt, R., Legendre, P., McGlinn, D., . . Wagner, H. (2018). vegan: Community Ecology Package.

Pages, F., Berger, A., Camus, M., Sanchez-Cabo, F., Costes, A., Molidor, R., ... Galon, J. (2005). Effector memory T cells, early metastasis, and survival in colorectal cancer. $N$ Engl J Med, 353(25), 2654-2666. doi:10.1056/NEJMoa051424

Pages, F., Kirilovsky, A., Mlecnik, B., Asslaber, M., Tosolini, M., Bindea, G., ... Galon, J. (2009). In situ cytotoxic and memory $\mathrm{T}$ cells predict outcome in patients with early-stage colorectal cancer. J Clin Oncol, 27(35), 5944-5951. doi:10.1200/JC0.2008.19.6147

Passardi, A., Canale, M., Valgiusti, M., \& Ulivi, P. (2017). Immune Checkpoints as a Target for Colorectal Cancer Treatment. International journal of molecular sciences, 18(6), 1324. doi:10.3390/ijms18061324

Pedregosa, F., Varoquaux, G., Gramfort, A., Michel, V., Thirion, B., Grisel, O., ... Duchesnay, E. (2011). Scikit-learn: Machine Learning in Python. Journal of Machine Learning Research, 12, 2825--2830.

Pinto, M. L., Rios, E., Duraes, C., Ribeiro, R., Machado, J. C., Mantovani, A., ... Oliveira, M. J. (2019). The Two Faces of Tumor-Associated Macrophages and Their Clinical Significance in Colorectal Cancer. Front Immunol, 10, 1875. doi:10.3389/fimmu.2019.01875

Popovic, G. C., Warton, D. I., Thomson, F. J., Hui, F. K. C., \& Moles, A. T. (2019). Untangling direct species associations from indirect mediator species effects with graphical models. Methods in Ecology and Evolution, 10(9), 1571-1583. doi:10.1111/2041210X.13247

Rosenthal, R., Cadieux, E. L., Salgado, R., Bakir, M. A., Moore, D. A., Hiley, C. T., ... consortium, T. R. (2019). Neoantigen-directed immune escape in lung cancer evolution. Nature, 567(7749), 479-485. doi:10.1038/s41586-019-1032-7

Schenck, R. O., Lakatos, E., Gatenbee, C., Graham, T. A., \& Anderson, A. R. A. (2019). NeoPredPipe: high-throughput neoantigen prediction and recognition potential pipeline. BMC Bioinformatics, 20(1), 264. doi:10.1186/s12859-019-2876-4

Schreiber, R. D., Old, L. J., \& Smyth, M. J. (2011). Cancer immunoediting: integrating immunity's roles in cancer suppression and promotion. Science, 331(6024), 15651570. doi:10.1126/science.1203486

Seshan, V. E. (2018). clinfun: Clinical Trial Design and Data Analysis Functions. 
Sethi, G., Sung, B., \& Aggarwal, B. B. (2008). TNF: a master switch for inflammation to cancer. Front Biosci, 13, 5094-5107. doi:10.2741/3066

Shukla, S. A., Rooney, M. S., Rajasagi, M., Tiao, G., Dixon, P. M., Lawrence, M. S., ... Getz, G. (2015). Comprehensive analysis of cancer-associated somatic mutations in class I HLA genes. Nature biotechnology, 33(11), 1152--1158.

Sottoriva, A., Kang, H., Ma, Z., Graham, T. A., Salomon, M. P., Zhao, J., . . Curtis, C. (2015). A Big Bang model of human colorectal tumor growth. Nat Genet, 47(3), 209-216. doi:10.1038/ng.3214

Steidl, C., Lee, T., Shah, S. P., Farinha, P., Han, G., Nayar, T., .. . Gascoyne, R. D. (2010). Tumorassociated macrophages and survival in classic Hodgkin's lymphoma. $N$ Engl J Med, 362(10), 875-885. doi:10.1056/NEJMoa0905680

Strobl, M. A. R., West, J., Viossat, Y., Damaghi, M., Robertson-Tessi, M., Brown, J. S., ... . Anderson, A. R. A. (2020). Turnover modulates the need for a cost of resistance in adaptive therapy. Cancer Res. doi:10.1158/0008-5472.CAN-20-0806

Topalian, S. L., Drake, C. G., \& Pardoll, D. M. (2015). Immune checkpoint blockade: a common denominator approach to cancer therapy. Cancer Cell, 27(4), 450-461. doi:10.1016/j.ccell.2015.03.001

Traag, V. A., Waltman, L., \& van Eck, N. J. (2019). From Louvain to Leiden: guaranteeing well-connected communities. Sci Rep, 9(1), 5233. doi:10.1038/s41598-019-41695-Z

van Beers, E. H., Joosse, S. A., Ligtenberg, M. J., Fles, R., Hogervorst, F. B., Verhoef, S., \& Nederlof, P. M. (2006). A multiplex PCR predictor for aCGH success of FFPE samples. Br J Cancer, 94(2), 333-337. doi:10.1038/sj.bjc.6602889

Van der Auwera, G. A., Carneiro, M. O., Hartl, C., Poplin, R., del Angel, G., Levy-Moonshine, A., ... DePristo, M. A. (2013). From FastQ data to high confidence variant calls: the Genome Analysis Toolkit best practices pipeline. Current protocols in bioinformatics / editoral board, Andreas D. Baxevanis ... \$[\$et al.\$]\$, 11(1110), 11.10.11--11.10.33. doi:10.1002/0471250953.bi1110s43

Vinay, D. S., Ryan, E. P., Pawelec, G., Talib, W. H., Stagg, J., Elkord, E., .. Kwon, B. S. (2015). Immune evasion in cancer: Mechanistic basis and therapeutic strategies. Semin Cancer Biol, 35 Suppl, S185-S198. doi:10.1016/j.semcancer.2015.03.004

Walt, S. v. d., Schönberger, J. L., Nunez-Iglesias, J., Boulogne, F., Warner, J. D., Yager, N., . . . contributors, t. s.-i. (2014). scikit-image: Image processing in Python. PeerJ, 2(e453).

Wang, K., Li, M., \& Hakonarson, H. (2010). ANNOVAR: functional annotation of genetic variants from high-throughput sequencing data. Nucleic Acids Research, 38(16), e164--e164.

Wangersky, P. J. (1978). Lotka-Volterra Population Models. Annual Review of Ecology and Systematics, 9(1), 189-218. doi:10.1146/annurev.es.09.110178.001201

Werner, B., Case, J., Williams, M. J., Chkhaidze, K., Temko, D., Fernandez-Mateos, J., ... . Sottoriva, A. (2020). Measuring single cell divisions in human tissues from multiregion sequencing data. Nat Commun, 11(1), 1035. doi:10.1038/s41467-020-148446

Williams, M. J., Werner, B., Heide, T., Curtis, C., Barnes, C. P., Sottoriva, A., \& Graham, T. A. (2018). Quantification of subclonal selection in cancer from bulk sequencing data. Nat Genet, 50(6), 895-903. doi:10.1038/s41588-018-0128-6 
Wong, S. C., Puaux, A. L., Chittezhath, M., Shalova, I., Kajiji, T. S., Wang, X., ... Biswas, S. K. (2010). Macrophage polarization to a unique phenotype driven by B cells. Eur J Immunol, 40(8), 2296-2307. doi:10.1002/eji.200940288

Wynn, T. A., Chawla, A., \& Pollard, J. W. (2013). Macrophage biology in development, homeostasis and disease. Nature, 496(7446), 445-455. doi:10.1038/nature12034

Yu, P., Steel, J. C., Zhang, M., Morris, J. C., \& Waldmann, T. A. (2010). Simultaneous blockade of multiple immune system inhibitory checkpoints enhances antitumor activity mediated by interleukin-15 in a murine metastatic colon carcinoma model. Clin Cancer Res, 16(24), 6019-6028. doi:10.1158/1078-0432.CCR-10-1966

Zauber, A. G., Winawer, S. J., O'Brien, M. J., Lansdorp-Vogelaar, I., van Ballegooijen, M., Hankey, B. F., ... Waye, J. D. (2012). Colonoscopic polypectomy and long-term prevention of colorectal-cancer deaths. N Engl J Med, 366(8), 687-696. doi:10.1056/NEJMoa1100370 


\section{Supplemental Methods}

\section{Steady state analysis of deterministic mathematical model}

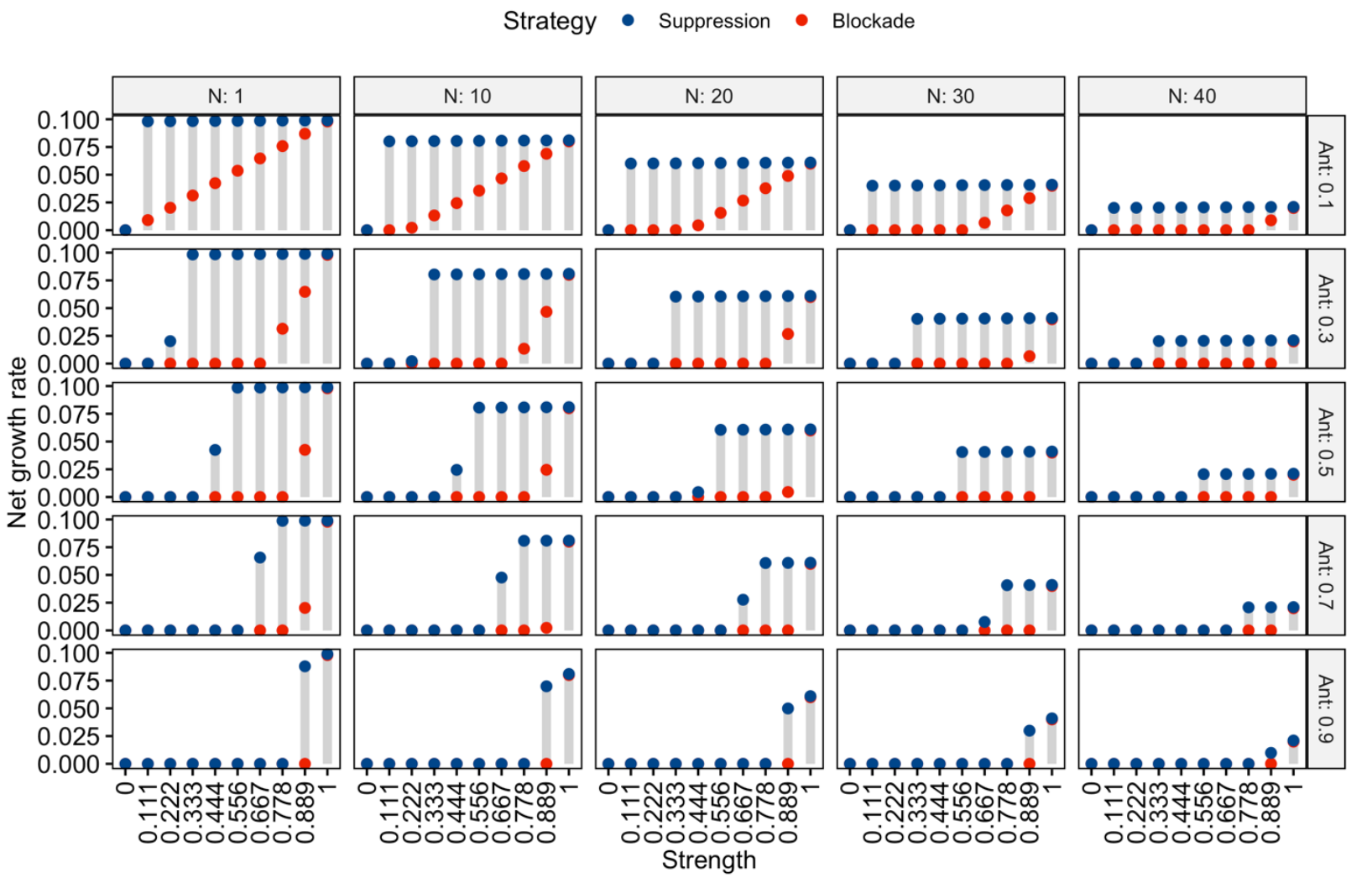

Figure S1. Here, we show the growth rate (i.e. the realized growth rate minus the kill and death rates $=\boldsymbol{r}_{i}\left(\frac{\boldsymbol{K}_{i}-\overbrace{\sum_{1}^{j} \alpha_{i j} N_{j}}+\overbrace{\boldsymbol{0 . 5}_{i}}^{\text {competition } \sigma_{i} N_{i}}}{K_{i}}\right)-$

$\gamma_{i} \overbrace{\left(\mathbf{1}-\frac{\sigma_{i}}{\gamma_{i}}\right)}^{\text {suppression }} \overbrace{\left(\mathbf{1}-\boldsymbol{\phi}_{i}\right)}^{\text {blockade }}-\boldsymbol{\delta}))$ for a new cell in a population in a larger population of $\mathrm{N}$ cells (columns) that has a carrying capacity $\mathrm{K}=100$. Each cell has an antigenicity (rows) and a different strength of Suppression $(0 \leq \sigma \leq 1, \phi=0)$ or Blockade $(\sigma=0,0 \leq \phi \leq 1)$. The Suppression strategy has a growth advantage over the Blockade strategy, giving it a selective advantage that allows it to outcompete Blockade. Furthermore, Suppression is more capable of invading populations, as it continues to have a positive net growth rate as the overall population approaches $\mathrm{K}$, whereas Blockade must be stronger to grow into larger populations.

In order to determine the long-term outcomes of the deterministic mathematical model we performed a linear steady state of the system in Equation 1. Given we were only interested in the outcome of the competition between two tumor subpopulations at a time (Get Lucky vs Get Smart; Suppression vs Blockade), we simplified the model to its 2-dimensional form, which can be rewritten as a Lotka-Volterra competition model. Subsequently, we used the well-known results about the linear stability of this system to classify the steady states (Gause-Witt isocline analysis (Gause \& Witt, 1935)) for details see e.g. (J. D. Murray, 2002). All calculations and plotting were carried out in R.

\section{Stochastic mathematical model}

A non-spatial agent-based model (ABM) was developed to simulate tumor evolution under immune predation and escape. Given the large population sizes, a branching process that produced millions of clones wasn't feasible, and so we limited the number of possible phenotypes. Each $i^{\text {th }}$ phenotype had 4 distinct traits: antigenicity $\left(\gamma_{i}\right)$, immune blockade $\left(\phi_{i}\right)$, immune suppression $\left(\sigma_{i}\right)$, and number of driver 
mutations $\left(n_{i}\right)$. Immune blockade/suppression traits were each binary, the maximum number of driver mutations was 4 , and there were 10,000 antigenicity values evenly distributed between $0-1$. Thus, in total, there were between and 50,000 - 200,000 unique phenotypes depending on immune suppression and blockade values. For example, if the immune suppression parameter was 0 , then there is only one immunosuppression phenotype, but if it is non-zero then there are 2 immunosuppression phenotypes ( 0 or $\sigma)$. We assumed that all phenotypes have the same intrinsic death rate, $\delta=0.01$.

The number of driver mutations, $n_{i}$, determined which "species" the phenotype belonged to, which in turn determined the phenotype's division rate $\left(r_{i}\right)$, carrying capacity $\left(K_{i}\right)$, and intra-species competition (found in the competition matrix, $\alpha$ ). If $n_{i}<2$, phenotype $i$ belonged to the epithelial species, which had a division rate of $r_{i}=0.2$ (Carulli, Samuelson, \& Schnell, 2014) and carrying capacity of $K_{i}=10^{7}$; if $2 \leq n_{i}<4$, the phenotype was of an adenoma, phenotype $i$ had a division rate of $r_{i}=0.2106$ and carrying capacity of $K_{i}=$ $10^{8}$; when $n \geq 4$ phenotype $i$ belonged to the carcinoma species and had division rate $r_{i}=0.221$ and carrying capacity $K_{i}=10^{9}$. Increases in division rates were based in differences in observed average KI67 quadrat density between CRA and CRC, which was 1.05 . This ratio was applied to the epithelial division rate, and then applied again to get the CRC division rate. The intra-species competition coefficients in $\alpha$ are based on the assumption that epithelial and adenomas occupy different areas, while carcinomas are invasive.

$\alpha=\begin{array}{cccc}E & 1 & A & C \\ A & 0 & 1 & 1 \\ C & 1 & 1 & 1\end{array}$

Each time step, a phenotype could create mutants, the number of which was determined by drawing from the binomial distribution, where the number of trials was the population size and the probability of success the cellular mutation rate $(\mu)$, i.e. $\operatorname{Binomial}\left(N=N_{i}, p=\mu\right)$. The cellular mutation rate of was calculated as follows. Exome size was estimated based on information in (M. Lek et al., 2016), which describes the exome as having $45 \mathrm{Mb}$, with $18 \mathrm{Mb}$ of possible synonymous variants. Therefore, we modeled the $45 \mathrm{Mb}-$ $18 \mathrm{Mb}=27 \mathrm{Mb}$ base pairs that may produce non-synonymous variants. The "normal" per division base pair mutation rates were set to 2.91 $\left(10^{-9}\right)$ (Monkol Lek et al., 2016; Werner et al., 2020). Given these values, we defined the cell mutation rate to be the probability of at least one non-synonymous mutation in the exome per cell per division: $0.0756=1-\operatorname{Binomial}\left(k=0, N=2.7 e 7, p=2.91\left(10^{-9}\right)\right)$.

We assume all mutations are inherited, and so all mutants have a higher antigenicity that their parent. In addition, mutants may also acquire one of three types of beneficial mutations: driver mutations, the ability to protect from T-cell attack, and the ability to recruit immunosuppressive cells. The multinomial distribution was used to determine how many of each mutant to make. Given that the number of genes in the genome has been estimated to be $g=20412$ (Cunningham et al., 2019), the probability of creating a cell that mutated an immunosuppressive gene is $\frac{1}{g}$. Likewise, the probability of creating a cell that mutated an immune blockade gene is also $\frac{1}{g}$.If there are 25 possible driver genes, then the probability a mutant has an additional driver mutation is $\frac{25-n_{i}}{g}$. Finally, the probability that the new cell didn't mutate any of these beneficial mutations, and only acquired a neoantigen, was $\frac{g-27+n_{i}}{g}$.

Therefore $\vec{m}=\operatorname{Multinomial}\left(N=u_{i j}^{t}, \vec{\mu}\right)$ is a four element vector where: $\vec{m}_{1}$ is the number of clones to create that do not have a beneficial mutation; $\vec{m}_{2}$ is the number of clones to create that acquire a driver mutation, with $m_{i j}$ being the number of driver mutations $N_{i j}$ has accumulated; $\vec{m}_{3}$ is the number of clones to create that express checkpoint inhibitors; and $\vec{m}_{4}$ is the number of clones to create that recruit immunosuppressive cells. The parameter $n$ is the number of genes in the genome, and $n_{\text {driver }}$ is the number of possible driver mutations.

The vector $\vec{u}$ contains the probability for each mutation type, with 


$$
\vec{u}=\left[1-\frac{n_{\text {driver }}-m_{i j}-2}{n}, \frac{n_{\text {driver }}-m_{i j}}{n}, \frac{1}{n}, \frac{1}{n}\right]
$$

Note that calculation of $\vec{m}_{2}$ assumes the number of driver mutations that will have an effect decreases as driver mutations are accumulated. In other words, it is assumed that if a driver is "hit" more than once, the benefit comes only with the first mutation, and subsequent hits have no effect. It is also assumed there is one gene for protecting from T-cell attack, and one gene for recruiting immunosuppressive cells.
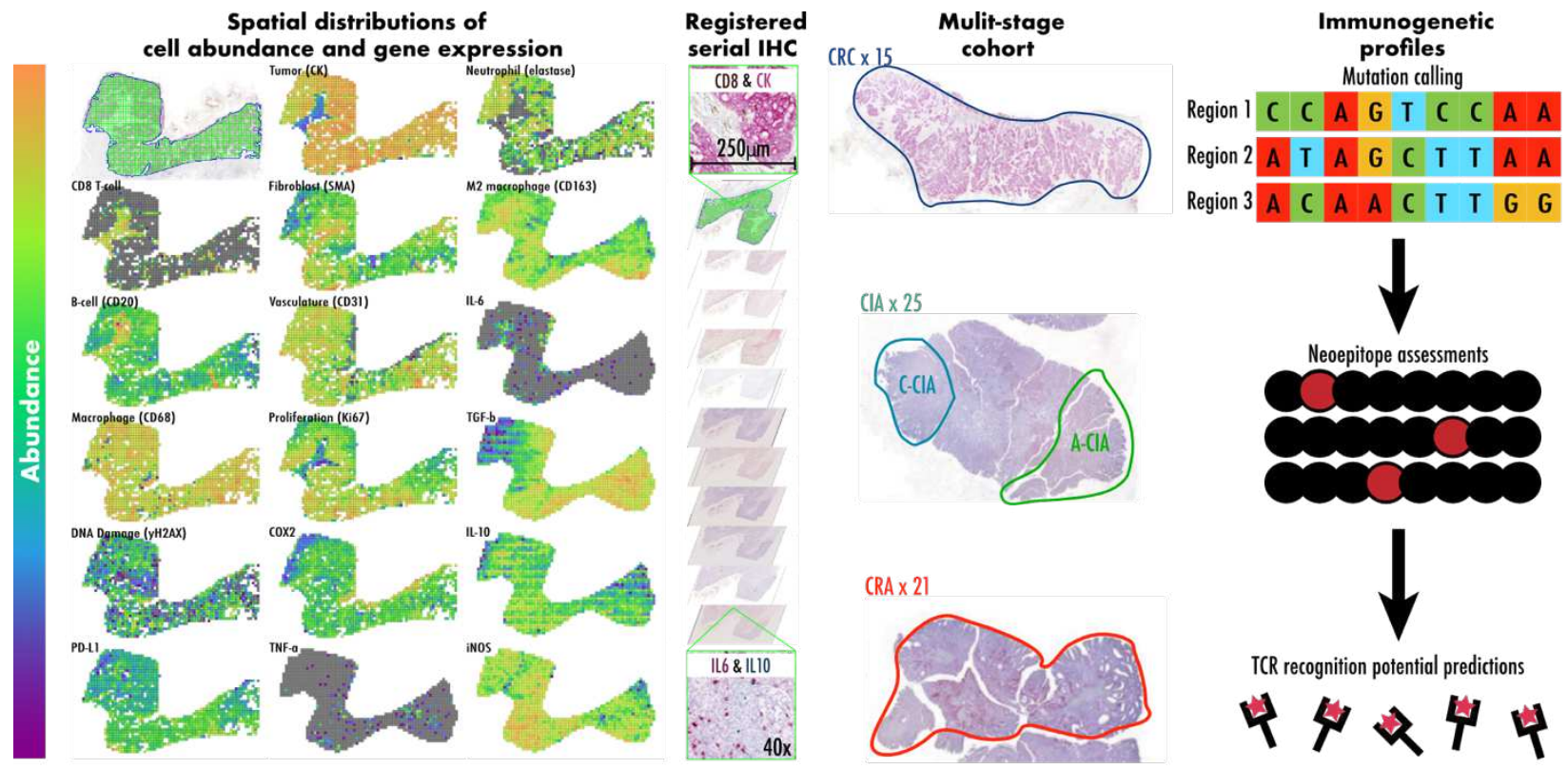

Figure S2. We studied changes in the tumor ecology and antigenic intra-tumor heterogeneity (alTH) using a cohort of 21 colorectal adenomas (CRA), 15 carcinomas (CRC), and 25 intermediate ca-in-ads (CIA), which have a carcinoma (C-CIA) emerging from its (presumably) ancestral adenoma (A-CIA). Given this unique dataset, we were able to determine what distinguishes benign adenomas (CRA) from those that progress (A-CIA), and what changes occur during progression from late adenoma ( $\mathrm{A}-\mathrm{CI}$ ) to nascent carcinoma (C-CIA) to mature carcinoma (CRC). Changes in the ecology were characterized using 17 cell markers and RNA transcripts associated with cytokine expression measured on whole slide images. Spatial analysis was conducted by registering the thin serial slices to create a spatially aligned composite image. The composite image was subsequently divided into $250 \mu \mathrm{m} \times 250 \mu \mathrm{m}$ quadrats, and abundance estimated as being proportional to the number of pixels positive for each marker at 40x magnification. Direct spatial associations were then quantified using the quadrat counts from the registered whole slide images, allowing us to determine colocalization of cell types and/or cytokine expression. alTH was quantified using multi-region whole exome sequencing followed by TCR recognition potential predictions. The results from this analysis allowed us to test the model's predictions about the timing and type of immune escape in colorectal cancer.

\section{Sample collection and processing}

FFPE samples $(n=54)$ representing adenomas (CRA, $n=13)$, adenomas with foci of cancer ('ca-in-ads', $\mathrm{CIA}, \mathrm{n}=24)$ and carcinomas $(\mathrm{CRC}, \mathrm{n}=17)$ were selected from the histopathology archives of University College Hospital, London, under UK ethical approval (07/Q1604/17) or John Radcliffe Hospital, Oxford under ethical approval (10/H0604/72). From each block, 7 serial sections were taken at 4 micron thickness, the first was stained with haematoxylin and eosin (H\&E) and used for histopathological classification by two expert pathologists (MRJ and MJ). The remaining 6 sections were used for dual color immunohistochemical staining. A further 6 sections at 5 micron were taken from a subset of blocks $(n=10)$, and used for DNA extraction.

\section{DNA extraction}


Different histopathological regions within individual lesions were demarcated on H\&E slides by an experienced $\mathrm{GI}$ pathologist. This was used to guide careful needle dissection and DNA was then extracted from these discrete areas using DNA QIAamp Mini Kit (Qiagen) and standard protocols.

\section{Whole exome sequencing}

Multi-region whole exome sequencing (WES) was performed on a subset of samples representing CRA ( $\mathrm{n}$ $=4$ with two regions each), $\mathrm{CIAs}(\mathrm{n}=3$, with one region from the carcinoma region and two from the adenoma region) and $\mathrm{CRC}(\mathrm{n}=3$, with two regions each). The quality of extracted FFPE DNA was verified using a multiplex PCR as previously described (van Beers et al., 2006) and only DNA samples that showed successful amplification of fragments greater than $300 \mathrm{bp}$ in length were considered for WES. DNA input of 50ng was used to prepare sequencing libraries with the Nextera Rapid Capture Exome kit, according to manufacturer's instructions (Illumina, Cambridge, UK). Libraries were sequenced with a target depth of $60 x$ on Illumina's HiSeq 2500 with 125 bp paired end reads ( $\mathrm{v} 4$ chemistry). Additional samples were added from (Sottoriva et al., 2015) adding to the total number of multi-region CRA and CRC ( $n=3$ and $n=3$, respectively). Alignments to the hg19 reference genome were conducted using the BWA-mem algorithm (Li \& Durbin, 2010) and processed using the GATK best practices workflow (Van der Auwera et al., 2013) for downstream analysis.

\section{Variant Calling}

Prior to calling variants, we normalized binary alignment/map (BAM) by down sampling to the lowest observed average depth across samples. First we calculated the average depth for target capture regions using samtools (Li et al., 2009). Once the average depth was calculated for each sample, the proportion of reads needed for each sample to reach the minimum average depth was calculated. Down-sampling was then performed using this proportion for each BAM file using PICARD (Broad Institute, 2017). This was conducted on each sample's region to generate ten replicate BAM files. Variant calling was then performed for each replicate sample group (multiple regions against normal) using multiSNV (Josephidou, Lynch, \& Tavaré, 2015), a joint calling method specifically designed for multi-region same patient experimental designs. Criteria used for assessing variant candidates dictated a minimum mapping quality of 30 , a minimum base quality of 20 , with at least five reads in the tumor and normal regions, and two variant alleles. Once a variant call set was obtained variants were further scrutinized for additional criteria. A total of ten reads were required within all normal sites and variant sites with no variant alleles present in the normal. Furthermore, a variant must be supported by a minimum number of two variant reads for at least one region; while the minimum variant allele frequency in one region is 0.1 .

\section{Neoantigen Predictions}

Human leukocyte antigen (HLA) haplotypes (A, B, and C) were called using PolySolver (Shukla et al., 2015) prior to down sampling on all normal regions for each patient. Neoantigen predictions were performed using NeoPredPipe (Schenck, Lakatos, Gatenbee, Graham, \& Anderson, 2019), which utilizes ANNOVAR (Wang, Li, \& Hakonarson, 2010) for variant annotations and NetMHCpan (Jurtz et al., 2017), NeoPredPipe is specifically designed to handle multi-region sequence samples. Only MHC-class I neoantigens were assessed for peptides of 8,9 , and $10-\mathrm{kmer}$ lengths. A minimum cut-off of $500 \mathrm{nM}$ binding affinities were used to be considered a putative neoantigen. To assess T-cell receptor binding potential an implementation of Łuksza et al.'s (Łuksza et al., 2017) recognition potential algorithm implemented within NeoPredPipe was used. 

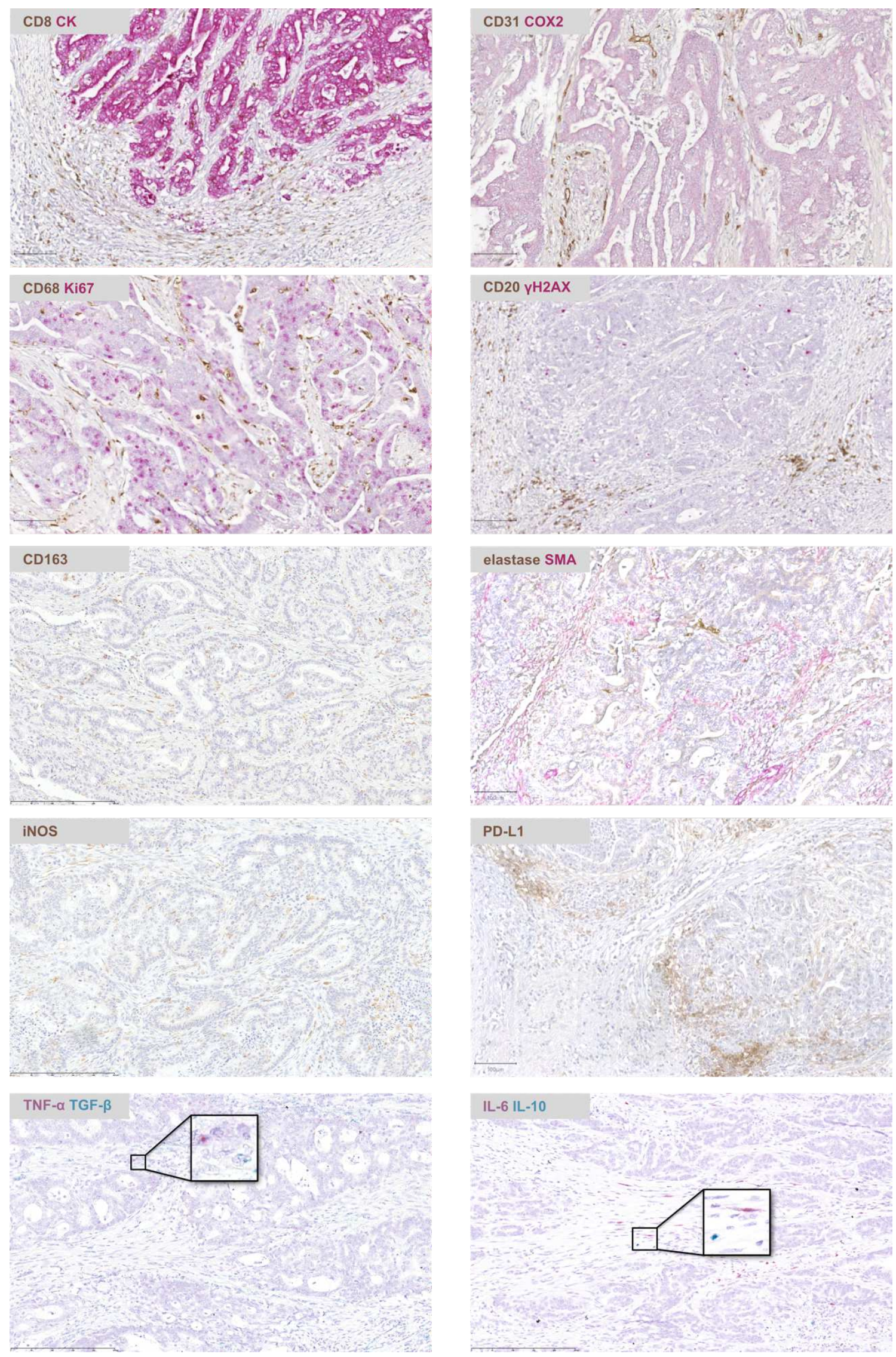
Figure S3 The tumor immune ecology was described using 17 markers to identify tumor cells (CK), cytotoxic T-cells (CD8), macrophages (CD68), M2 macrophages (CD163), M1 macrophages (iNOS), neutrophils (elastase), B-cells (CD20), PD-L1, DNA damage ( $\mathrm{YH} 2 \mathrm{AX}$ ), proliferation (Ki67), inflammatory cytokines (TNF- $\alpha, \mathrm{IL}-6)$, and immunosuppressive cytokines (TGF- $\beta$, IL-10). These $10 x$ images come from a CRC, but the analysis was conducted at $40 x$.

\section{Dual color immunohistochemistry (IHC)}

Sequential dual color IHC of 10 markers was performed according to standard protocol. Briefly, $4 \mu \mathrm{m}$ serial sections were dewaxed, rehydrated and immersed in 3\% hydrogen peroxide for 20 minutes to quench endogenous peroxidase activity. Antigen retrieval was carried out at $95^{\circ} \mathrm{C}$ for 20 minutes in sodium citrate buffer ( $\mathrm{pH}$ 6.0), unless otherwise specified (Supplementary Table 2). After cooling, sections were incubated with blocking buffer (PBS supplemented with $2 \%$ goat serum and $1 \%$ bovine serum albumin) for 1 hour at RT. Primary antibodies were diluted in blocking buffer and applied for 1 hour at RT or overnight at 4oC (see Supplementary Table 2 for antibody details). Sections were then incubated with a biotinylated secondary antibody at RT for 45 min, followed by incubation with streptavidin-biotin peroxidase solution at RT for 45 min. Visualization of the first antibody binding was carried out using DAB, according to the manufacturer's instructions (Vector Labs, Peterborough, UK). Slides then underwent a second round of antigen retrieval, generally at $95^{\circ} \mathrm{C}$ for 5 minutes in sodium citrate buffer ( $\left.\mathrm{pH} 6.0\right)$, before applying the blocking buffer for a further 1 hours at RT. The second primary antibody was then applied (see Supplementary Table 2 for details), followed by incubation with a biotinylated secondary antibody at RT for 45 min and incubation with streptavidin-alkaline phosphatase. Visualization of the second antibody binding was performed using Fast Red, according to the manufacturer's instructions (Abcam, Cambridge, UK). Finally, sections were lightly counterstained using Gill's haematoxylin, and allowed to dry before mounting and digitizing using the Pannoramic 250 high throughput scanner (3D Histech, Budapest, Hungary).

\section{In situ hybridization (ISH)}

Dual-color RNA ISH was performed to detect the expression of TGF $\beta$, TNFa, IL6 and IL10 using commercially available reagents (Advanced Cell Diagnostics, Newark, CA). The RNAscope $2.5 \mathrm{HD}$ Duplex Reagent Kit (catologue number 322430) was used according to the manufacturer's instructions with the probes Hs-TGFB1 (400881), Hs-TNFA-C2 (310421-C2), Hs-IL10 (602051) and Hs-IL6-C2 (310371-C2).

\section{Computational image analysis}

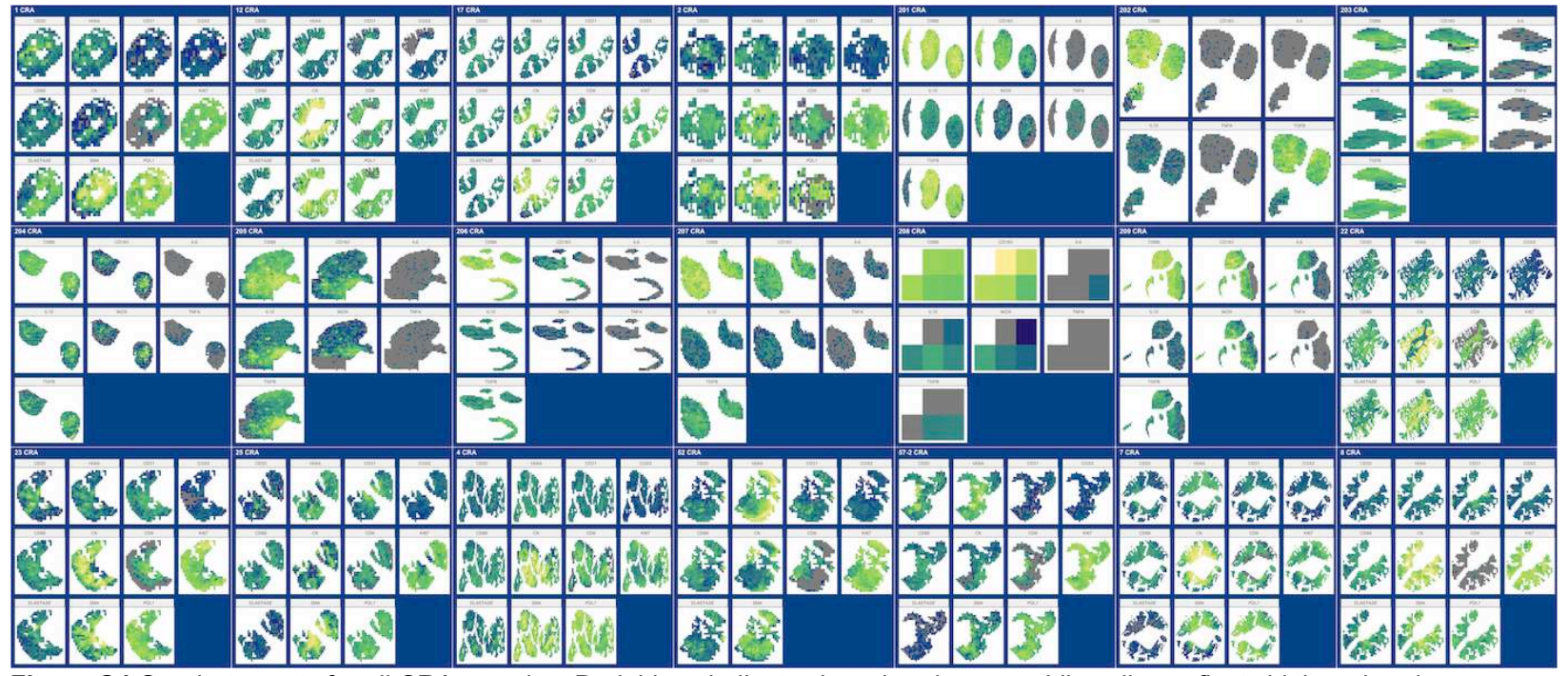

Figure S4 Quadrat counts for all CRA samples. Dark blues indicates low abundances, while yellow reflects higher abundances. 


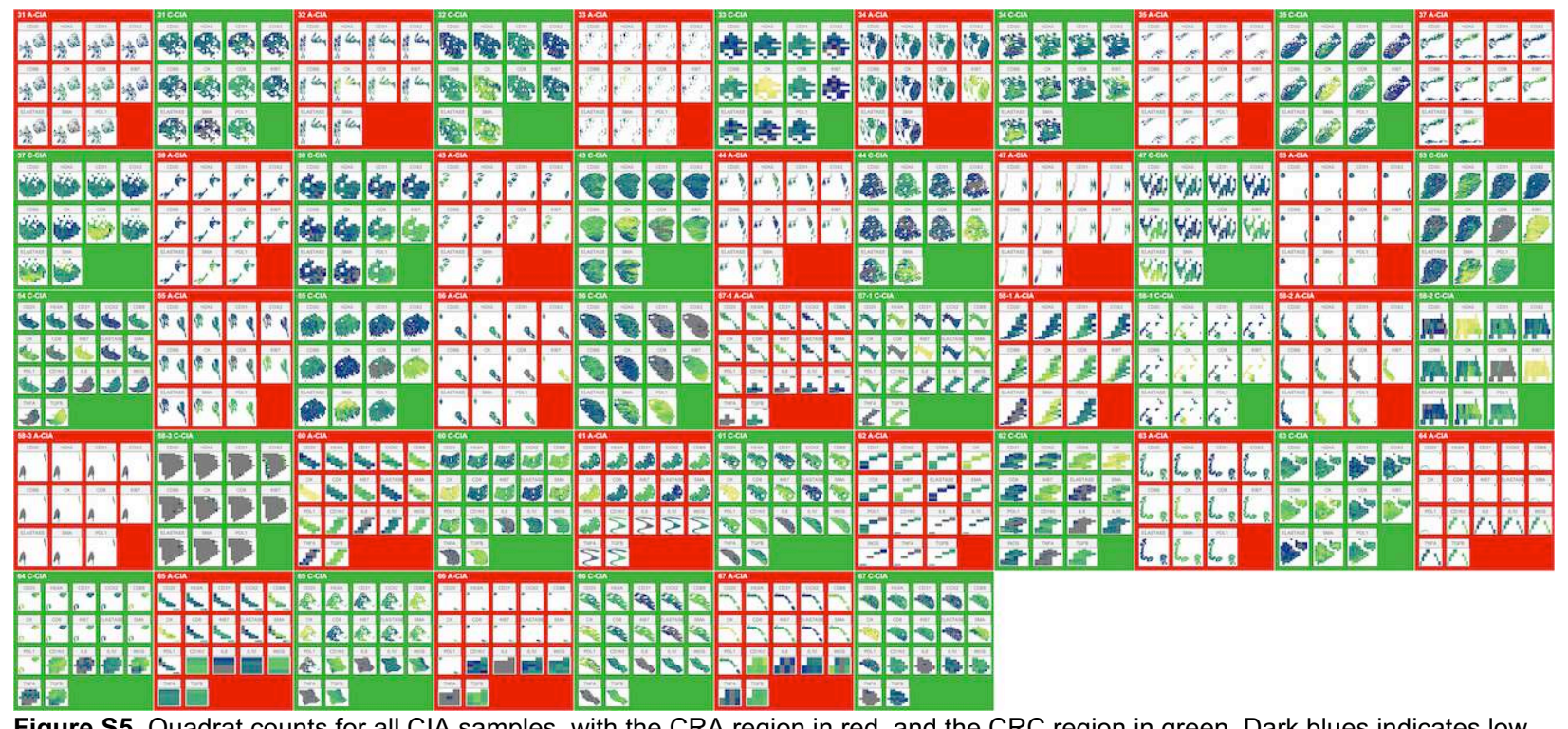

Figure S5. Quadrat counts for all CIA samples, with the CRA region in red, and the CRC region in green. Dark blues indicates low abundances, while yellow reflects higher abundances.

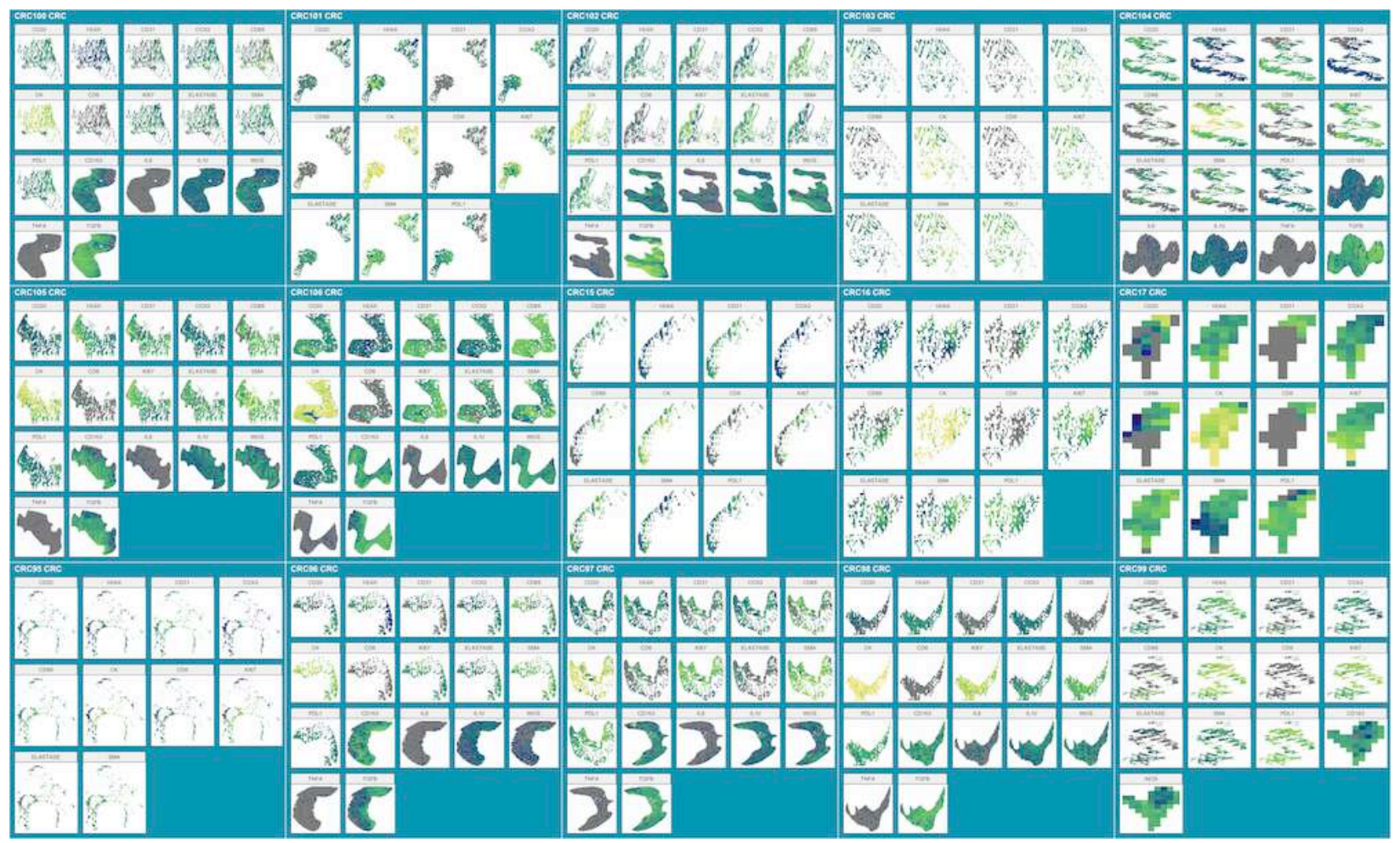

Figure S6. Quadrat counts for all CRC samples. Dark blues indicates low abundances, while yellow reflects higher abundances.

All image processing was conducted using OpenCV (Bradski, 2000), scikit-image (Walt et al., 2014), scikit-learn (Pedregosa et al., 2011; Schreiber et al., 2011), and OpenSlide (Goode, Gilbert, Harkes, Jukic, \& Satyanarayanan, 2013) for the Python programming language. This research utilized Queen Mary's Apocrita HPC facility, supported by QMUL Research-IT (King, Butcher, \& Zalewski, March, 2017). 


\section{Whole Slide Image Registration}

The collection of transformation matrices to align serial slices was found using virtual alignment of pathology image series (VALIS) software (in prep). Briefly, this method determines the optimal order in which to align the images, and then finds the rigid transformation matrices to align each image the stack to the previous image in the stack. It uses KAZE feature detection (Alcantarilla, Bartoli, \& Davison, 2012), brute force matching, and RANSAC filtering (Fischler \& Bolles, 1981) to get the points used in the estimation of the transformation matrices. The images used in this step of registration were resized to be $<1000$ pixels in both dimensions.

Quadrat corners, each 250 microns apart, were determined using the dimensions of a full-size reference image, such as the CD8/CK slide. The quadrat positions were then scaled down to fit within the image used during registration. Using the transformation matrices, the quadrat positions where then moved up/down the stack, warped to the unaligned image, and then rescaled for the full-size image. A mask was also used to create a tissue outline, and only quadrats that were completely within the mask were used.

\section{Stain segmentation}

The stains in each quadrat were separated using the following methods. In the first set of samples, three individuals (A.M.B, M.P.N., S.Y.H) conducted the staining, thus there was inter-batch variability and no single approach worked well for all. Thus, we trained a support vector machine (SVM) for each individual's collection of stained images. Four features were used to train and predict: the pixel's RGB values, and descriptor (SCD) (Khan, Rajpoot, Treanor, \& Magee, 2014). In all cases, the accuracy of each SVM was above $90 \%$. A final step to correct for misclassifications was to convert the each segmented image to HSV colorspace, and then reclassify brown pixels as red if their $\mathrm{H}>=100$, and red pixels as brown if $4<\mathrm{H}<$ 100. This algorithm used OpenCV (Bradski, 2000)colorspace conversion.

In the second sample set, all samples stained by the same person (A.M.B.), and the following method was used to separate red, brown, and green. The images were converted to cylindrical LAB colorspace (LCh) and saturation (S) calculated as the chroma normalized by lightness. All channels were then standardized to be in the range of 0-255. A pixel was considered green if all the following were true: $\mathrm{h}<$ 50 or $h>250, S>=30$. Likewise, a pixel was positive for red if $90<=h<145, S>200$. A pixel was classified as brown if $145<=h<210$ and $S>100$. In this algorithm, scikit-image (Walt et al., 2014) was used to convert the RGB image to LAB.

\section{Ecological Image Analysis}




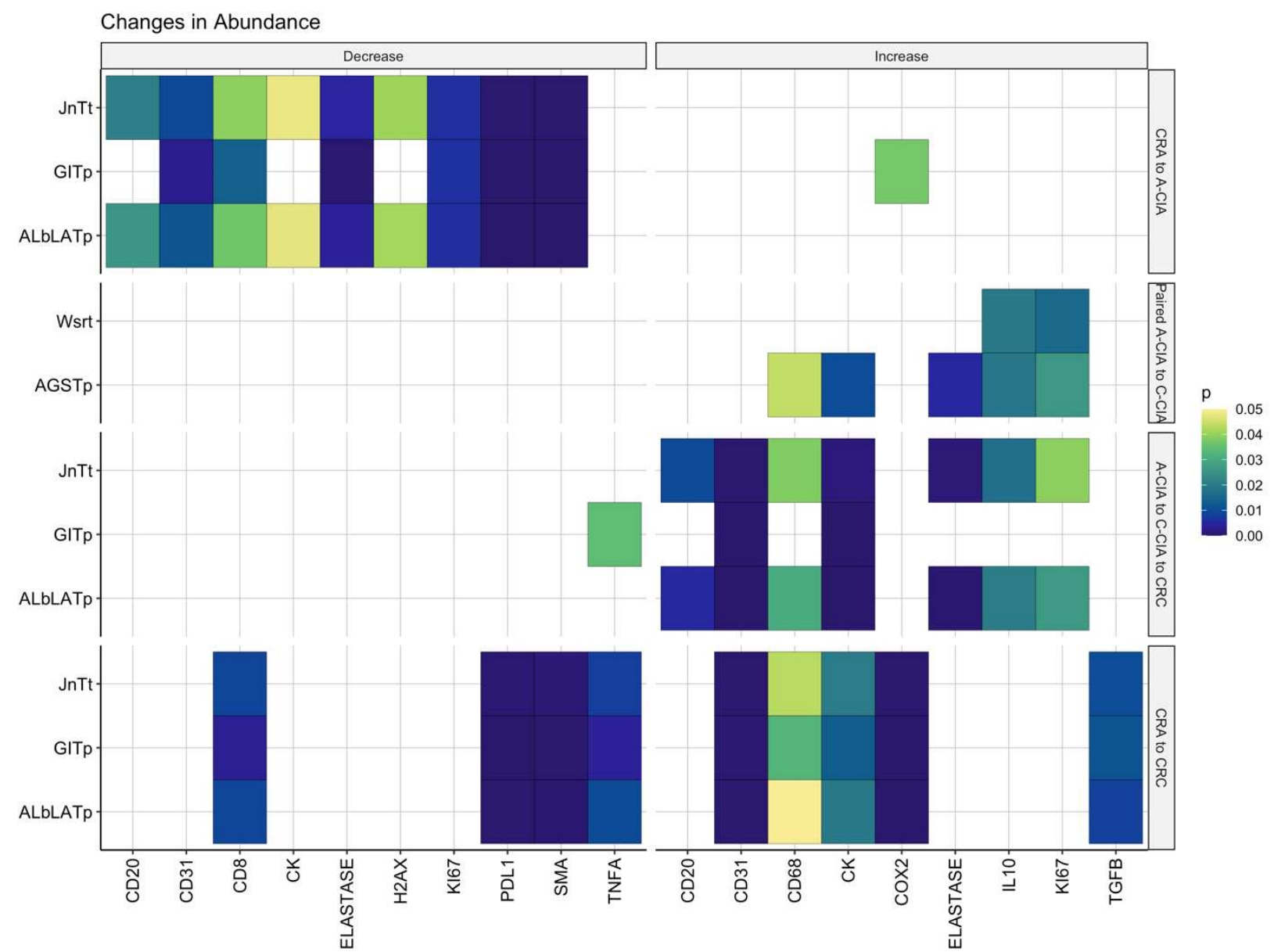

Figure S7. Significance tests for changes in abundance. A combination of frequentist statistics (y-axis) were used to determine if there were significant directional changes, where "Decrease" means there was a decrease in the abundance of a marker ( $\mathrm{x}$-axis) in each type of comparison. For example, all tests found a significant decrease in CD8 when comparing A-CIA to C-CIA to CRC.

Paired tests were used to determine how abundances changed in the same tumor, from the A-CIA to its neighboring C-CIA. Colored tiles show significance, whereas missing tiles indicate non-significance. . KWrst= Kruskal-Wallis rank sum test,

AKWTp=Approximative Kruskal-Wallis Test (permutation), GITp= General Independence Test (permutation), ALbLATp= Approximative Linear-by-Linear Association Test (permutation), $\mathrm{JnTt}=$ Jonckheere-Terpstra test.

Description of the tumor ecology within and across various stages was accomplished using a variety of methods. Cell abundances (assumed to be proportional to the number of pixels positive for each stain) were measured from the registered images, using the averaged quadrat counts (assumed to be proportional to the number of positive pixels) to determine if there were directional changes over time, i.e. if there were significant increases or decreases in abundance during progression from late adenoma to mature carcinoma. The significance of these trends was determined using a combination of frequentist and permutation statistical tests (Figures S7 and S9). A similar analysis was conducted to determine if there were directional changes in spatial associations between the various cell types. Quadrat counts were used to construct a species association network (Popovic et al., 2019), whose coefficients were compared across stages. In addition to testing for these inter-stage trends, paired tests were used to determine if there were significant intra-tumor changes in cell abundance and interactions. This was accomplished by comparing the carcinoma $(\mathrm{C}-\mathrm{CIA})$ to its precursor adenoma $(\mathrm{A}-\mathrm{CI} A)$ in the same $\mathrm{CIA}$ sample.

Several ecological tests were used to compare tumor-immune ecologies across stages. We quantified and compared the amount of ecological homogeneity within each tumor stage, using multivariate homogeneity of group dispersions (PERMDISP2)(M. J. Anderson, 2006). Permutational multivariate analysis of variance (PERMANOVA) was used to determine if there were significant differences in the structure of the ecological communities of each tumor stage, which was then visualized using constrained analysis of principal coordinates (CAP) (Marti J. Anderson \& Willis, 2003; Oksanen et al., 2018). Using indicator species analysis, we determined which, if any, cell types define the different tumor stages (De Cáceres \& 
Legendre, 2009). Finally, the Mantel test was used to determine if differences in the immune ecology and microenvironment are correlated (Legendre \& Legendre, 2012), as might be expected to occur during tumor instigated immune remodeling.

A distance matrix, which compares tumors based on the whole collection of markers, is required for many of the ecological tests we performed. We tested all combinations of distance metrics and normalization methods in the vegan $\mathrm{R}$ package (Oksanen et al., 2018) and found that the Jaccard distance on log-scaled PPC most often had adjacent adenomas in CIA samples as being the most similar to one another, as would be expected. It should be noted that the Jaccard distance in the vegan package is not the same as a traditional Jaccard distance, but more of a variant of the Bray-Curtis distance. Thus, we performed the analysis on log-scaled data and a distance matrix constructed using the Jaccard distance. PERMANOVA, PERMDSIP2, CAP, and the Mantel tests were all conducted on this distance matrix.

Tumor-immune ecological dissimilarities

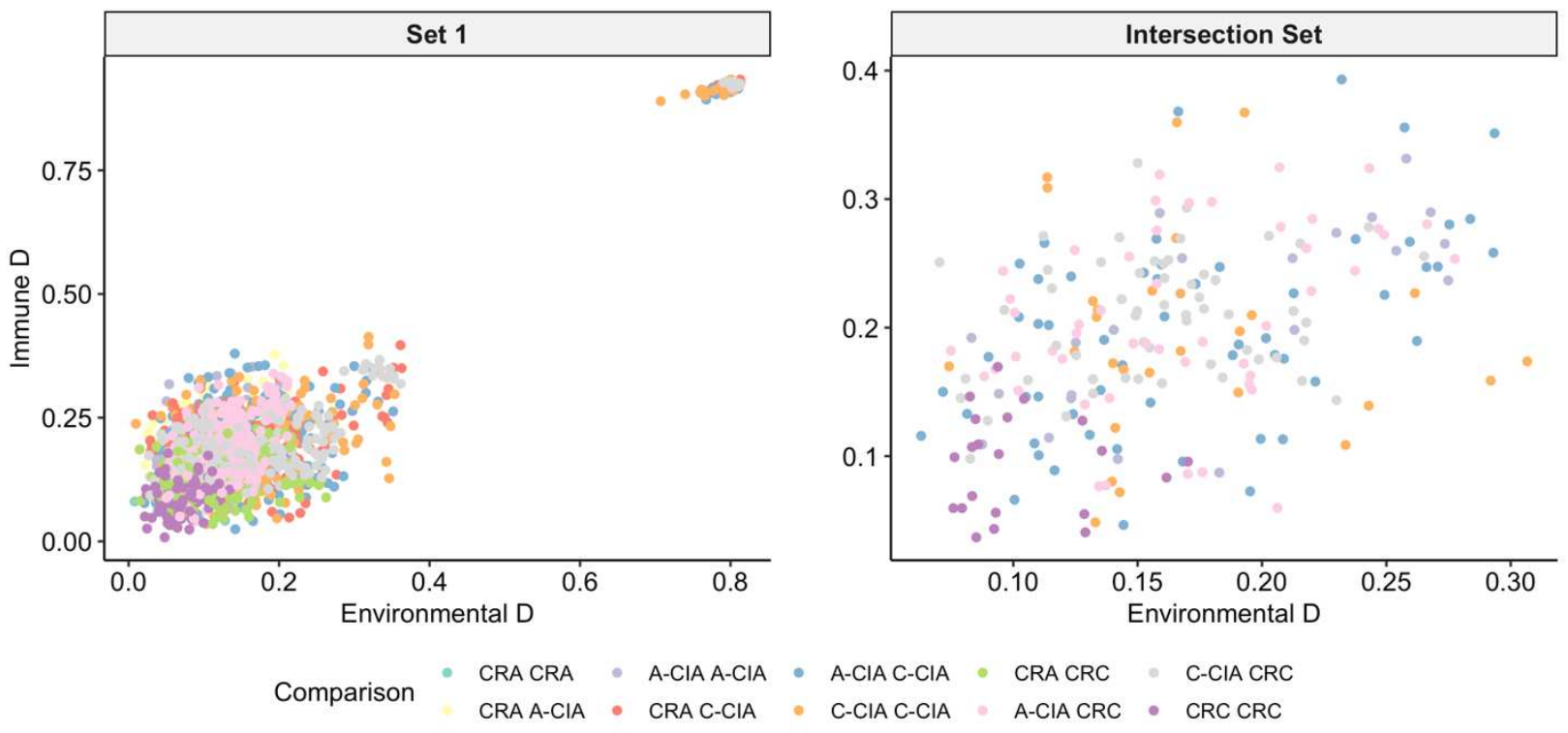

Figure S8 Differences in immune composition (cytotoxic T-cells, B-cells, macrophages, and neutrophils) and environment (tumor cells, vasculature, PDL1, and fibroblasts) are significantly correlated, as determined by the Mantel test. This indicates the immune system and tumor microenvironment change together, consistent with a scenario in which tumors create an immunosuppressive niche that further promotes tumor growth. 


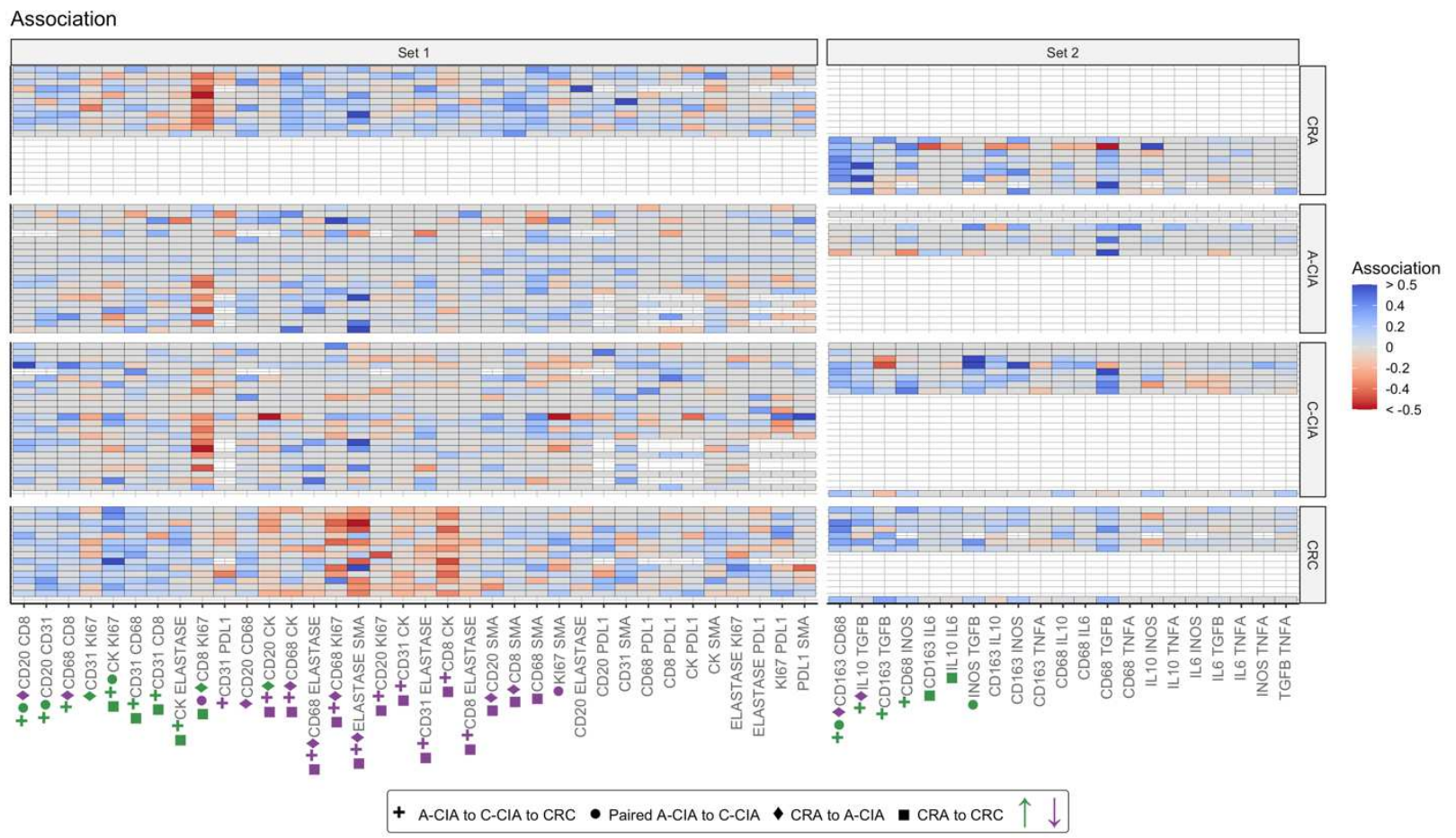

Figure S9. Table showing significant changes in direct pairwise cell-cell/cell-cytokine spatial associations, where each row is a sample and each column a spatial association. Shapes below each marker pair signify that there were significant changes in spatial association across the specified group. Green indicates that the association increased from the first group to the last group, while purple indicates that the spatial association decreased. Here, surprisingly, we observed that, compared to A-CIA, CRA have a higher association of CD68/CD163 (purple diamond), indicative of M2 macrophages, and a greater association between TGF- $\beta$ \& IL-10, suggesting CRA are more immunosuppressive than A-CIA. Interestingly, the same trend of increased CD68/CD163 and IL-10/ TGF$\beta$ are observed during the evolution of the tumor from progressed adenoma to carcinoma. A notable difference is that CD8 more strongly spatially co-localized with tumor (CK) in CRA compared to A-CIA and CRC (purple diamond and square), and the association between CD8 and tumor decreases from progressed adenoma to CRC (purple plus). This suggests that while CRA may have some immunosuppressive cells and cytokines, they remain most immunogenic. 


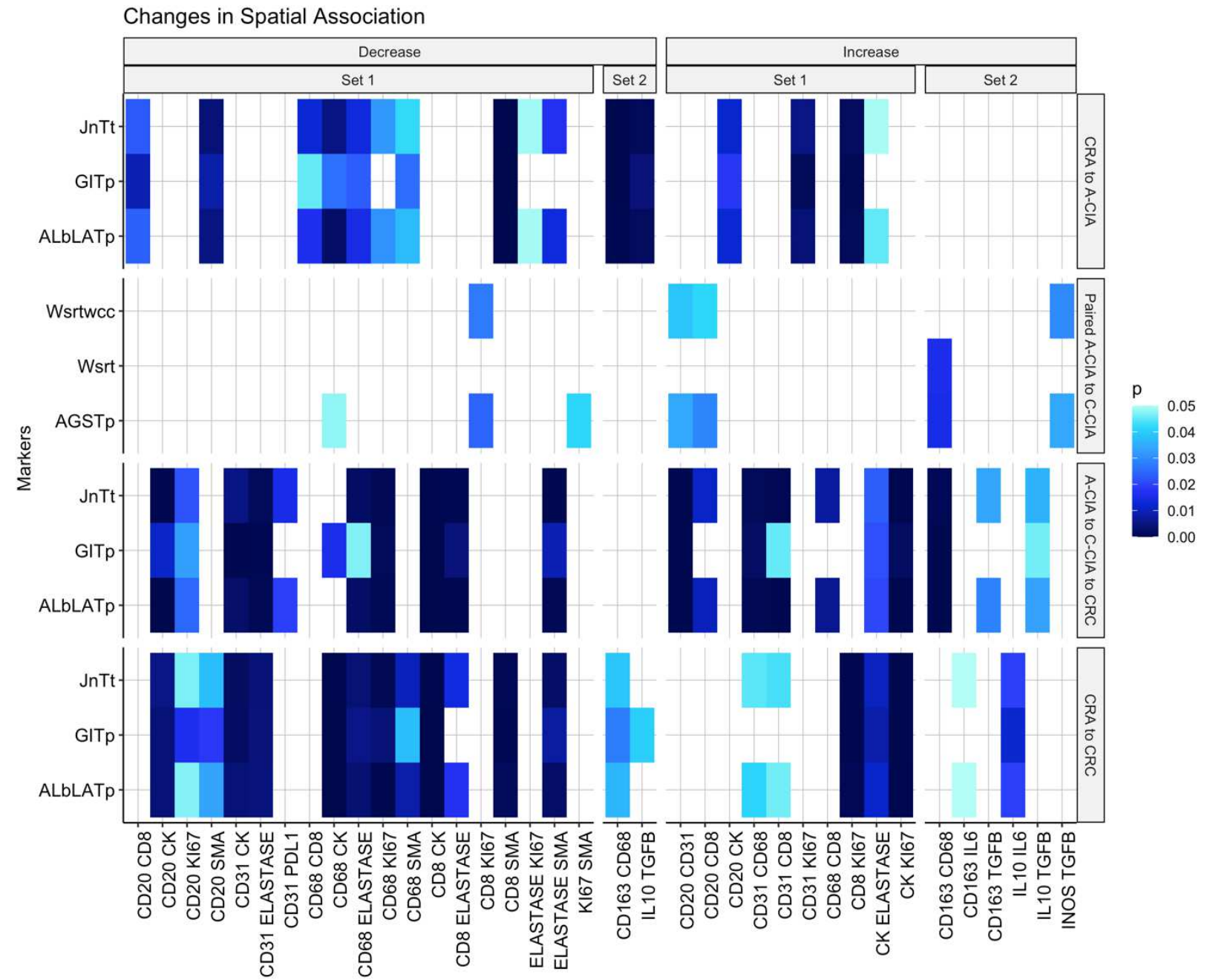

Figure S10. Significance tests for changes in spatial associations. A combination of frequentist statistics (y-axis) were used to determine if there were significant directional changes, where "Decrease" means there was a decrease in the abundance of a marker (x-axis) in each type of comparison. For example, all tests found a significant decrease in the spatial association between $\mathrm{CD} 8$ and $\mathrm{CK}$ when comparing $\mathrm{A}-\mathrm{CIA}$ to $\mathrm{C}-\mathrm{CIA}$ to $\mathrm{CRC}$. Paired tests were used to determine how abundances changed in the same tumor, from the $\mathrm{A}-\mathrm{CIA}$ to its neighboring $\mathrm{C}-\mathrm{ClA}$. Colored tiles show significance, whereas missing tiles indicate non-significance. KWrst $=$ Kruskal-Wallis rank sum test, AKWTp=Approximative Kruskal-Wallis Test (permutation), GITp= General Independence Test (permutation), ALbLATp= Approximative Linear-by-Linear Association Test (permutation), JnTt= Jonckheere-Terpstra test.

A suite of statistical tests was used to detect significant changes in marker positivity, co-localization, and direct cell-cell interactions (Figures S7 and S9). Several methods were used to find those results that were robust, as those that were found to be significant across multiple tests are most likely to be true. The twosided Kruskal-Wallis rank sum test, two-sided approximate Kruskal-Wallis Test, and two-sided General Independence Test were used to determine there were differences between the tumor subtypes CRA, A$\mathrm{CIA}, \mathrm{C}-\mathrm{CIA}$, and CRC. Assuming that the carcinomas will take over the adenomas in the ca-in-ad samples, and thus develop into carcinomas, one can test for trends in the data by setting the timing order of A-CIA$>\mathrm{C}$-CIA -> CRC. Having ordered the subtypes, one-sided Approximative Linear-by-Linear Association Test and the Jonckheere-Terpstra test were used to determine if there a significant increase or decrease across groups (Seshan, 2018). The Conover-Iman test of multiple comparisons using rank sums was used as the post-hoc test to determine which subtypes were significantly different from one another (Dinno, 2017). 
The paired-tests looking for differences between A-CIA and C-CIA included the General Symmetry test and the Wilcoxon rank sum test. As with the tests comparing all subtypes, the two-sided version of these tests was used to detect differences, and the one-sided test was used to detect the presence of trends and the direction of those trends.

The two-sided approximate Kruskal-Wallis Test, General Independence Test, General Symmetry test, and Approximative Linear-by-Linear Association Tests are permutation tests and were conducted using the coin package for R (Hothorn, Hornik, Wiel, \& Zeileis, 2008).

\section{Model Fitting}
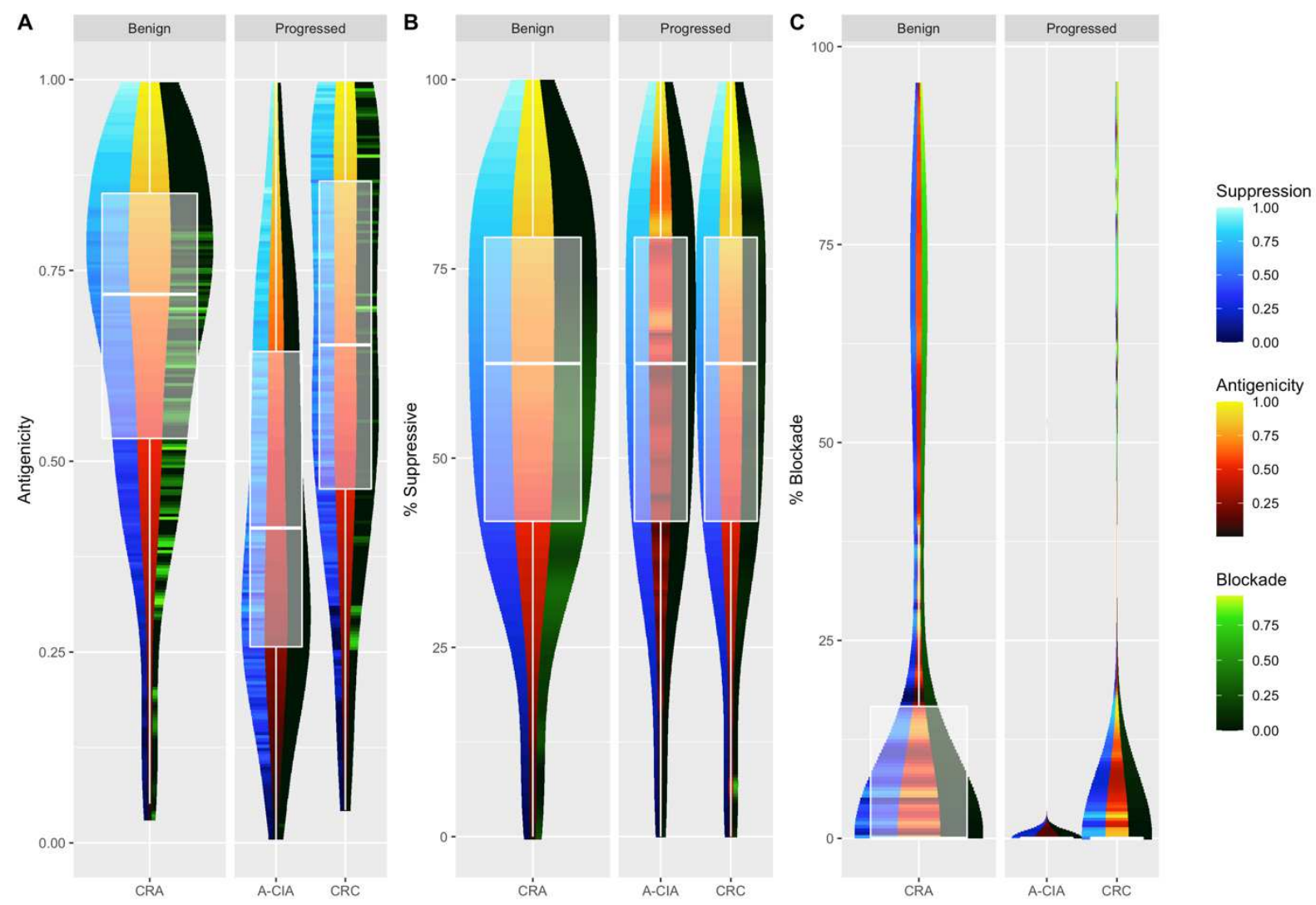

Figure S11 Distribution of antigenicities (A), and percent of the tumor with immunosuppressive cell infiltrate (B), and percent of the tumor with blockade (PD-L1) (C), in benign adenomas compared to those of progressed adenomas and carcinomas using the subset of parameters that matched the data. The horizontal bin in each violin is colored by the average value of phenotypes that are within that bin. For each parameter combination that reproduced the observed patterns, the two most different malignant and benign tumors were selected for plotting.

\section{Supplementary Materials}

\section{Supplementary table 1 - Sample details}




\begin{tabular}{|c|c|c|c|c|}
\hline $\begin{array}{l}\text { Sample } \\
\text { number }\end{array}$ & Classification & Site & Used for WES & Used for IHC \\
\hline 1 & Adenoma & Oxford & & $\mathrm{Y}$ \\
\hline 2 & Adenoma & Oxford & & $\mathrm{Y}$ \\
\hline 4 & Adenoma & Oxford & $\mathrm{Y}$ & $\mathrm{Y}$ \\
\hline 6 & Adenoma & Oxford & $\mathrm{Y}$ & \\
\hline 7 & Adenoma & Oxford & & $Y$ \\
\hline 8 & Adenoma & Oxford & & $Y$ \\
\hline 12 & Adenoma & Oxford & $\mathrm{Y}$ & $\mathrm{Y}$ \\
\hline 17 & Adenoma & Oxford & & $\mathrm{Y}$ \\
\hline 22 & Adenoma & Oxford & $\mathrm{Y}$ & Y \\
\hline 23 & Adenoma & Oxford & & $\mathrm{Y}$ \\
\hline 25 & Adenoma & Oxford & & $\mathrm{Y}$ \\
\hline 52 & Adenoma & $\mathrm{UCH}$ & & $\mathrm{Y}$ \\
\hline $57-2$ & Adenoma & $\mathrm{UCH}$ & & $\mathrm{Y}$ \\
\hline K & Adenoma & & $\mathrm{Y}$ & \\
\hline $\mathbf{S}$ & Adenoma & & $Y$ & \\
\hline $\mathbf{P}$ & Adenoma & & $Y$ & \\
\hline 31 & Ca-in-ad & Oxford & & $\mathrm{Y}$ \\
\hline 32 & Ca-in-ad & Oxford & $\mathrm{Y}$ & $\mathrm{Y}$ \\
\hline 33 & Ca-in-ad & Oxford & $\mathrm{Y}$ & $\mathrm{Y}$ \\
\hline 34 & Ca-in-ad & Oxford & & $\mathrm{Y}$ \\
\hline 35 & Ca-in-ad & Oxford & & $\mathrm{Y}$ \\
\hline 37 & Ca-in-ad & Oxford & & $\mathrm{Y}$ \\
\hline
\end{tabular}




\begin{tabular}{|c|c|c|c|c|}
\hline 38 & Ca-in-ad & Oxford & & $\mathrm{Y}$ \\
\hline 43 & Ca-in-ad & Oxford & & $\mathrm{Y}$ \\
\hline 44 & Ca-in-ad & Oxford & & $\mathrm{Y}$ \\
\hline 47 & Ca-in-ad & Oxford & Y & $\mathrm{Y}$ \\
\hline 53 & Ca-in-ad & $\mathrm{UCH}$ & & $\mathrm{Y}$ \\
\hline 55 & Ca-in-ad & $\mathrm{UCH}$ & & $\mathrm{Y}$ \\
\hline 56 & Ca-in-ad & $\mathrm{UCH}$ & & $Y$ \\
\hline $57-1$ & Ca-in-ad & $\mathrm{UCH}$ & & $\mathrm{Y}$ \\
\hline $58-2$ & Ca-in-ad & $\mathrm{UCH}$ & & $\mathrm{Y}$ \\
\hline $58-3$ & Ca-in-ad & $\mathrm{UCH}$ & & $\mathrm{Y}$ \\
\hline 60 & Ca-in-ad & $\mathrm{UCH}$ & & $\mathrm{Y}$ \\
\hline 61 & Ca-in-ad & $\mathrm{UCH}$ & & $\mathrm{Y}$ \\
\hline 62 & Ca-in-ad & $\mathrm{UCH}$ & & $\mathrm{Y}$ \\
\hline 63 & Ca-in-ad & $\mathrm{UCH}$ & & $\mathrm{Y}$ \\
\hline 64 & Ca-in-ad & $\mathrm{UCH}$ & & $\mathrm{Y}$ \\
\hline 65 & Ca-in-ad & $\mathrm{UCH}$ & & $\mathrm{Y}$ \\
\hline 66 & Ca-in-ad & $\mathrm{UCH}$ & & $\mathrm{Y}$ \\
\hline 67 & Ca-in-ad & $\mathrm{UCH}$ & & $\mathrm{Y}$ \\
\hline 15 & Carcinoma & Oxford & $\mathrm{Y}$ & $\mathrm{Y}$ \\
\hline 16 & Carcinoma & Oxford & $\mathrm{Y}$ & $\mathrm{Y}$ \\
\hline 17 & Carcinoma & Oxford & $\mathrm{Y}$ & $\mathrm{Y}$ \\
\hline 54 & Carcinoma & $\mathrm{UCH}$ & & $\mathrm{Y}$ \\
\hline $58-1$ & Carcinoma & $\mathrm{UCH}$ & & $\mathrm{Y}$ \\
\hline 95 & Carcinoma & $\mathrm{UCH}$ & & $\mathrm{Y}$ \\
\hline 96 & Carcinoma & $\mathrm{UCH}$ & & $\mathrm{Y}$ \\
\hline 97 & Carcinoma & $\mathrm{UCH}$ & & $\mathrm{Y}$ \\
\hline 98 & Carcinoma & $\mathrm{UCH}$ & & $Y$ \\
\hline
\end{tabular}




\begin{tabular}{|l|l|l|l|l|}
\hline $\mathbf{9 9}$ & Carcinoma & $\mathrm{UCH}$ & \\
\hline $\mathbf{1 0 0}$ & Carcinoma & $\mathrm{UCH}$ & & $\mathrm{Y}$ \\
\hline $\mathbf{1 0 1}$ & Carcinoma & $\mathrm{UCH}$ & $\mathrm{Y}$ \\
\hline $\mathbf{1 0 2}$ & Carcinoma & $\mathrm{UCH}$ & $\mathrm{Y}$ \\
\hline $\mathbf{1 0 3}$ & Carcinoma & $\mathrm{UCH}$ & $\mathrm{Y}$ \\
\hline $\mathbf{1 0 4}$ & Carcinoma & $\mathrm{UCH}$ & \\
\hline $\mathbf{1 0 5}$ & Carcinoma & $\mathrm{UCH}$ & & $\mathrm{Y}$ \\
\hline $\mathbf{1 0 6}$ & Carcinoma & $\mathrm{UCH}$ & & $\mathrm{Y}$ \\
\hline $\mathbf{M}$ & Carcinoma & & & \\
\hline $\mathbf{N}$ & Carcinoma & & $\mathrm{Y}$ & \\
\hline $\mathbf{T}$ & Carcinoma & & $\mathrm{Y}$ & \\
\hline $\mathbf{G}$ & Carcinoma & & $\mathrm{Y}$ & \\
\hline $\mathbf{W}$ & Carcinoma & & $\mathrm{Y}$ & \\
\hline & & & & \\
\hline
\end{tabular}

Supplementary table 2 - Antibody details

\begin{tabular}{|l|l|l|l|l|}
\hline $\begin{array}{l}\text { Slide no. } \\
\text { (antibody no.) }\end{array}$ & Antibody & $\begin{array}{l}\text { Manufacturer } \\
\text { (cat no.) }\end{array}$ & Dilution & $\begin{array}{l}\text { Antigen } \\
\text { Retrieval }\end{array}$ \\
\hline $\mathbf{1}(\mathbf{1})$ & $\begin{array}{l}\text { Monoclonal } \\
\text { mouse anti- } \\
\text { human CD8 }\end{array}$ & Dako (M7103) & $1: 100$ & $\begin{array}{l}\text { Sodium citrate, } \\
\text { pH } 6.0\end{array}$ \\
\hline
\end{tabular}




\begin{tabular}{|c|c|c|c|c|}
\hline $1(2)$ & $\begin{array}{l}\text { Monoclonal } \\
\text { mouse anti- } \\
\text { human } \\
\text { cytokeratin }\end{array}$ & Dako (M3515) & $\begin{array}{l}\text { 1:100 } \\
\text { 1hr RT }\end{array}$ & $\begin{array}{l}\text { Sodium citrate, } \\
\mathrm{pH} 6.0\end{array}$ \\
\hline $2(1)$ & $\begin{array}{l}\text { Monoclonal } \\
\text { mouse anti- } \\
\text { human CD20 }\end{array}$ & Dako (M0755) & $\begin{array}{l}\text { 1:300 } \\
\text { 1hr RT }\end{array}$ & $\begin{array}{l}\text { Sodium citrate, } \\
\mathrm{pH} 6.0\end{array}$ \\
\hline $2(2)$ & $\begin{array}{l}\text { Monoclonal } \\
\text { mouse anti- } \\
\text { human } \mathrm{YH} 2 \mathrm{AX}\end{array}$ & $\begin{array}{l}\text { Abcam } \\
\text { (ab26350) }\end{array}$ & $\begin{array}{l}\text { 1:500 } \\
\text { 1hr RT }\end{array}$ & $\begin{array}{l}\text { Sodium citrate, } \\
\mathrm{pH} 6.0\end{array}$ \\
\hline $3(1)$ & $\begin{array}{l}\text { Monoclonal } \\
\text { mouse anti- } \\
\text { human CD68 }\end{array}$ & Dako (M0876) & $\begin{array}{l}\text { 1:100 } \\
\text { 1hr RT }\end{array}$ & $\begin{array}{l}\text { Sodium citrate, } \\
\mathrm{pH} 6.0\end{array}$ \\
\hline $3(2)$ & $\begin{array}{l}\text { Monoclonal } \\
\text { rabbit anti- } \\
\text { human Ki67 }\end{array}$ & $\begin{array}{l}\text { Abcam } \\
\text { (ab16667) }\end{array}$ & $\begin{array}{l}1: 2000 \\
1 \mathrm{hr} \mathrm{RT}\end{array}$ & $\begin{array}{l}\text { Sodium citrate, } \\
\mathrm{pH} 6.0\end{array}$ \\
\hline $4(1)$ & $\begin{array}{l}\text { Monoclonal } \\
\text { mouse anti- } \\
\text { human } \\
\text { neutrophil } \\
\text { elastase }\end{array}$ & Dako (M0752) & $\begin{array}{l}\text { 1:20 } \\
\text { 1hr RT }\end{array}$ & None \\
\hline $4(2)$ & $\begin{array}{l}\text { Monoclonal } \\
\text { mouse anti- } \alpha- \\
\text { smooth muscle } \\
\text { actin (SMA) }\end{array}$ & Sigma (A2547) & $\begin{array}{l}\text { 1:3000 } \\
\text { 1hr RT }\end{array}$ & $\begin{array}{l}\text { Sodium citrate, } \\
\mathrm{pH} 6.0\end{array}$ \\
\hline $5(1)$ & $\begin{array}{l}\text { Monoclonal } \\
\text { mouse anti- } \\
\text { human CD31 }\end{array}$ & $\begin{array}{l}\text { Novocastra } \\
\text { (CD31-1A10) }\end{array}$ & $\begin{array}{l}1: 100 \\
\mathrm{O} / \mathrm{N} 4^{\circ} \mathrm{C}\end{array}$ & $\begin{array}{l}\text { Sodium citrate, } \\
\mathrm{pH} 6.0\end{array}$ \\
\hline $6(1)$ & $\begin{array}{l}\text { Monoclonal } \\
\text { rabbit anti- } \\
\text { human PD-L1 }\end{array}$ & $\begin{array}{l}\text { Cell Signaling } \\
\text { Technology } \\
\text { (E1L3N) }\end{array}$ & $\begin{array}{l}\text { 1:400 } \\
\text { 1hr RT }\end{array}$ & $\begin{array}{l}\text { Tris-EDTA, pH } \\
9.0\end{array}$ \\
\hline Set 2 & $\begin{array}{l}\text { Polyclonal } \\
\text { rabbit anti-iNOS }\end{array}$ & $\begin{array}{l}\text { Abcam } \\
\text { (ab15323) }\end{array}$ & $\begin{array}{l}\text { 1:100 } \\
\text { 1hr RT }\end{array}$ & $\begin{array}{l}\text { Tris-EDTA, pH } \\
9.0\end{array}$ \\
\hline Set 2 & $\begin{array}{l}\text { Monoclonal } \\
\text { mouse anti- } \\
\text { CD163 }\end{array}$ & $\begin{array}{l}\text { Novus (NB110- } \\
59935)\end{array}$ & $\begin{array}{l}\text { 1:100 } \\
\text { 1hr RT }\end{array}$ & $\begin{array}{l}\text { Tris-EDTA, pH } \\
9.0\end{array}$ \\
\hline
\end{tabular}




\begin{tabular}{|l|l|l|l|l|}
\hline- & $\begin{array}{l}\text { Polyclonal goat } \\
\text { anti-rabbit IgG } \\
\text { biotinylated }\end{array}$ & Dako (E0432) & $1: 400$ & - \\
\hline$-\quad$ & $\begin{array}{l}\text { Polyclonal } \\
\text { rabbit anti- } \\
\text { mouse IgG } \\
\text { biotinylated }\end{array}$ & Dako (E0354) & $1: 400$ & - \\
\hline $\begin{array}{l}\text { Streptavidin- } \\
\text { HRP }\end{array}$ & Dako (P0397) & $1: 500$ & - \\
\hline$-\quad$ Streptavidin-AP & $\begin{array}{l}\text { Life } \\
\text { Technologies } \\
\text { (S921) }\end{array}$ & $\begin{array}{l}1: 500 \\
45 \mathrm{~min} \text { RT }\end{array}$ & - \\
\hline$-\quad$ & $45 \mathrm{~min}$ RT & \\
\hline
\end{tabular}

